\title{
An analytical model of ground surface vibration due to axisymmetric wave motion in buried fluid-filled pipes
}

\author{
Yan Gao $^{a *}$, Jennifer M. Muggleton ${ }^{b}$, Yuyou Liu ${ }^{c, d}$, Emiliano Rustighi $^{b}$ \\ ${ }^{a}$ Key Laboratory of Noise and Vibration Research, Institute of Acoustics, Chinese Academy of \\ Sciences, Beijing 100190 China \\ ${ }^{b}$ Institute of Sound and Vibration Research, University of Southampton, Highfield, \\ Southampton SO17 1BJ UK \\ ${ }^{c}$ Beijing Municipal Institute of Labour Protection, Beijing 100054 China \\ ${ }^{d}$ AECOM Infrastructure \& Environment UK Limited, London SW19 4DR UK
}

Abstract: The axisymmetric $(n=0)$ fluid-borne $(s=1)$ wave has been exploited with varying degrees of success in practical surveys for determining the location of buried pipes. Difficulties are sometimes encountered in interpreting ground surface vibration data, whilst attempting to locate the pipes, due to the occurrence of abrupt changes in the phase response over the usable frequency range. Based on a wave propagation model developed recently, this paper presents an analytical model for predicting the ground surface displacements resulting from the radiated elastic waves in the soil medium. Two representative soils have been specifically considered, where the $s=1$ wave in the pipe will leak shear waves into the soil, but may or may not leak compressional waves. In each of these cases, numerical simulations are presented to predict the ground surface displacements. The model is used to demonstrate how, when both compressional and shear waves are radiated, they can interfere such that abrupt phase changes occur at the frequencies coincident with magnitude minima in the ground surface displacements; when only shear waves are radiated, such interference does not occur. Furthermore, for sandy soil, it is found that the horizontal displacement is dominated by the radiated shear wavenumber component whereas the vertical displacement is controlled by the radiated compressional wavenumber component. Using the analytical 
1 model, theoretical predictions of ground surface displacements are compared with

2 experimental data from a dedicated MDPE pipe rig.

3 Key words: buried pipes; fluid-borne wave; elastic waves; ground surface vibration.

4 *Corresponding author. E-mail address: gaoyan@mail.ioa.ac.cn

5 1. Introduction

6 Buried pipelines are central to modern life and form an important part of many

7 engineering structures for transporting fluids and gases. Pipe location is a subject of

8 increasing concern in China and across the world due to ever increasing congestion in our

9 underground space, combined with the need to continually maintain, repair and replace assets

10 within that space. It is becoming apparent that utility maps often contain inaccurate data and,

11 moreover, are invariably incomplete. With this in mind, a general urban survey is currently

12 underway in China to gather the first authentically recorded location information about

13 existing pipes laid underground [1]. In other countries too, considerable effort is underway to

14 rectify these issues [2].

15 The way in which axisymmetric $(n=0)$ waves propagate in fluid-filled pipes has been the 16 subject of numerous studies [3-9]. Fuller and Fahy [3] investigated the dispersion behaviour 17 and energy distributions of free waves in thin-walled fluid-filled pipes; Pinnington and 18 Briscoe [4], in particular, focused on axisymmetric $(n=0)$ wave motion within the fluid-filled 19 pipes in vасио. When the pipe is surrounded by an elastic medium, the vibrational energy is 20 transmitted along the pipe in a variety of modes, and can couple energy into the surrounding 21 medium due to the leaky modes leaving the pipe wall [5, 6]. More recently, the present 22 authors [7-9] have developed models for the study of the propagation characteristics of the 23 fluid-borne $(s=1)$ wave within fluid-filled pipes surrounded by an elastic medium and the 24 torsional $(s=0)$ wave, all of which may radiate to the ground surface. These cited works 25 suggests that well below the pipe ring frequency (usually at least $1 \mathrm{kHz}$ ), the $s=1$ wave is 
1 often the main carrier of vibrational energy within oil- or water-filled piping systems. This is

2 in marked contrast to the case of the pipe in an air medium in which the excitation of the

3 bending mode contributes mostly, in particular at lower frequencies [10]. The low-frequency

$4 \quad s=1$ wave propagates predominantly within the contained fluid but is accompanied by some

5 radial pipe wall motion. Furthermore, it has been demonstrated experimentally in an earlier

6 study that this wave can effectively radiate into the surrounding soil and be detected at the

7 ground surface; in this case axial phase dependence mirrors that in the pipe [11]. Using this

8 knowledge an acoustic technique has been developed for the detection of underground pipes:

9 the pipe is intentionally and directly excited at some known location (e.g. via a hydrant)

10 aboveground with concurrent vibrational mapping of the ground surface, in order to infer the

11 location of the remainder of the pipe. (It is possible that excitation of the ground in the

12 vicinity of the pipe could also elicit waves in the pipe but such an arrangement would be by

13 no means optimal.) It was shown that the phase of the ground vibration data can be exploited

14 to reveal the pipe location, with the magnitude data providing a useful supplement in 15 identifying discontinuities (such as leaks). However, it was noticed that, although the spatial 16 phase dependence at ground surface matched that in the pipe, the phase variation with 17 frequency at a single location above the pipe contained abrupt phase changes which could not 18 be properly accounted for, as illustrated in Fig. 1. Since this first study, it has been found that 19 these phase phenomena commonly occur and, to date, no satisfactory explanation for the 20 occurrences has been offered.

21 The low-frequency propagation characteristics of the $s=1$ wave have received much attention in the literature and wave equations presented in previous research; however, the surrounding spaces (be they fluid or elastic) have, in general, been restricted to infinite ones.

24 The presence of a free surface, or indeed any other discontinuity in the soil elastic properties, will affect the form of wave solutions. To date, there has only been a limited amount of work 
1 carried out into the study of radiated elastic waves in the surrounding soil itself and effects of

2 the ground surface. Jette and Parker [12] first investigated the ground surface displacements

3 accompanying the propagation of acoustic waves in a buried gas-filled pipe. As evidenced by

4 the experimental data, the ground surface vibration, in response to the internal acoustic

5 pressure, was found to be predominantly governed by the axisymmetric vibration of the pipe

6 at frequencies ranging from $200 \mathrm{~Hz}$ to $2 \mathrm{kHz}$. Numerical methods, for example the finite

7 element method, have been developed to investigate the vibrational behaviour of cylindrical

8 underground structures in an elastic half-space in seismic applications [13-17]. Although they

9 have shown to be effective for the analysis of the propagation of cylindrical waves associated

10 with energy radiation, the accurate modelling of the 3D dynamic structure becomes

11 impossible in practical situations due to the computational cost [17]. For ease of computation

12 as well as physical interpretation of wave phenomena, it is more desirable to explore the

13 analytical method for the study of the ground vibration response associated with wave

14 radiation due to pipe motion.

15 In this paper, a model is presented for predicting the ground surface displacements 16 resulting from the $s=1$ wave motion in a buried fluid-filled pipe. A comprehensive analysis of 17 the fully coupled system, including the ground surface, would be extremely complex and 18 beyond the scope of the present paper. Here a somewhat simplified analysis is presented, for 19 which the following assumptions are made [8]:

- The soil is assumed to be homogeneous and isotropic, resulting in only two types of body waves being present in the soil (a compressional wave and a shear wave) [18]. Other soil models do exist, such as the Biot model (and its modified versions) which consider soil porosity (see, for example $[19,20]$ ), and result in an increase in the number of possible waves in the soil, but here the aim is to use the simplest model which will still capture the salient features observed in practice and illuminate the 
dominant physical processes in play;

- The effects of the soil on the pipe and the effects of the waves propagating in the pipe on the soil can be considered independently; what this means in practice is that, in the calculation of the dispersion characteristics of the $s=1$ wave, the free ground surface (along with the concomitant wave reflections) is neglected - it is only included once the waves in the pipe have already, so to speak, been set up. Because of the large attenuation in most soils, this is only likely to become problematic at extremely low frequencies when the number of compressional/shear wavelengths between the pipe and the ground surface becomes very small;

- Once the waves radiating from the pipe reach the ground surface, they can be considered to be in the far field and undergo a plane wave treatment. This limits the lower frequency bound for which the analysis is valid, in a similar way to the assumption described above; Only response of the ground directly over the pipe is considered, for which only elastic body waves need to be considered.

The investigation starts with the introduction of the propagation and radiation of the $s=1$ wave confined to low frequencies, followed by a detailed description of the propagating wave motion in Section 2. The model is then incorporated into the analytical method to predict the ground surface displacements in Section 3. Section 4 presents some numerical results of the ground surface displacements for a PVC water pipe buried in two representative soils. General discussions follow on the predicted ground vibration response to explain the frequency-domain features, and to demonstrate the coherent interference of the conical compressional with shear waves in some sandy soils while being unlikely to happen in clay soils. Some experimental measurements are presented to support the analytical model in Section 5. Finally some conclusions are drawn in Section 6. 


\section{Fluid-borne wave motion}

Before considering how elastic waves radiate in the surrounding soil, the fluid-borne $(s=1)$ wave motion in a buried fluid-filled pipe needs to be studied. Based on our previous model to predict the dispersion relationship for the $s=1$ wave [7], this section investigates the soil vibration induced by the $s=1$ wave.

Consider a thin-walled fluid-filled pipe surrounded by an infinite elastic medium that can sustain elastic body waves, i.e., the compressional and shear waves as illustrated in Fig. 2. The pipe has a mean radius $a$ and wall thickness $h$. With reference to the cylindrical coordinate system, $u$ and $w$ denote the shell displacements in the $x$ and $r$ directions, respectively; and $u_{x}$ and $u_{r}$ denote the soil displacements. The surrounding soil is assumed to be elastic, homogenous and isotropic.

\subsection{Relationships between the internal pressure and the pipe wall displacements}

Consider the equations governing the coupled axial and radial motion for the axisymmetric $s$ waves in the buried fluid-filled pipe given in [7]

$$
\left[\begin{array}{cc}
\Omega^{2}-\left(k_{s} a\right)^{2}-S L_{11} & -\mathrm{i} v_{p}\left(k_{s} a\right)-S L_{12} \\
-\mathrm{i} v_{p}\left(k_{s} a\right)-S L_{21} & 1-\Omega^{2}-F L-S L_{22}
\end{array}\right]\left[\begin{array}{c}
U_{s} \\
W_{s}
\end{array}\right]=\mathbf{0}
$$

where $U_{s}$ and $W_{s}$ are the displacement amplitudes of the pipe wall; $\Omega$ is the non-dimensional frequency, $\Omega=k_{L} a ; k_{L}$ is the shell compressional wavenumber, $k_{L}^{2}=\omega^{2} \rho_{p}\left(1-v_{p}^{2}\right) / E_{p} ; \rho_{p}, E_{p}$ and $v_{p}$ are the density, Young's modulus and Poisson's ratio of the shell; fluid loading term, $F L$ and $\mathbf{S L}$ are the fluid loading term and the soil loading matrix representing the coupling effects of the fluid and soil respectively, and are given by

$$
F L=\frac{\rho_{f}}{\rho_{p}} \frac{a}{h} \frac{\Omega^{2}}{k_{f s}^{r} a} \frac{\mathrm{J}_{0}\left(k_{f s}^{r} a\right)}{\mathrm{J}_{0}^{\prime}\left(k_{f s}^{r} a\right)}
$$




$$
\begin{aligned}
& S L_{11}=-\mu_{m} \frac{\left(1-v_{p}^{2}\right)}{E_{p}} \frac{a}{h} \frac{k_{d s}^{r} a k_{r}^{2} a^{2}}{k_{r s}^{r} a k_{d s}^{r} a\left[\mathrm{H}_{0}\left(k_{r s}^{r} a\right) / \mathrm{H}_{0}{ }^{\prime}\left(k_{r s}^{r} a\right)\right]+k_{s}^{2} a^{2}\left[\mathrm{H}_{0}\left(k_{d s}^{r} a\right) / \mathrm{H}_{0}{ }^{\prime}\left(k_{d s}^{r} a\right)\right]} \\
& \left.S L_{12}=\mathrm{i} \mu_{m} \frac{\left(1-v_{p}^{2}\right)}{E_{p}} \frac{a}{h} k_{s} a\left\{2-\frac{k_{r}^{2} a^{2} \mathrm{H}_{0}\left(k_{d s}^{r} a\right) / \mathrm{H}_{0}{ }^{\prime}\left(k_{d s}^{r} a\right)}{k_{r s}^{r} a k_{d s}^{r} a\left[\mathrm{H}_{0}\left(k_{r s}^{r} a\right) / \mathrm{H}_{0}{ }^{\prime}\left(k_{r s}^{r} a\right)\right]+k_{s}^{2} a^{2}\left[\mathrm{H}_{0}\left(k_{d s}^{r} a\right) / \mathrm{H}_{0}^{\prime}\left(k_{d s}^{r} a\right)\right]}\right\}\right\} \\
& S L_{21}=S L_{12} \\
& S L_{22}=-\mu_{m} \frac{\left(1-v_{p}^{2}\right)}{E_{p}} \frac{a}{h}\left\{2+\frac{k_{r s}^{r} a k_{r}^{2} a^{2}\left[\mathrm{H}_{0}\left(k_{r s}^{r} a\right) / \mathrm{H}_{0}{ }^{\prime}\left(k_{r s}^{r} a\right)\right]\left[\mathrm{H}_{0}\left(k_{d s}^{r} a\right) / \mathrm{H}_{0}{ }^{\prime}\left(k_{d s}^{r} a\right)\right]}{k_{r s}^{r} a k_{d s}^{r} a\left[\mathrm{H}_{0}\left(k_{r s}^{r} a\right) / \mathrm{H}_{0}{ }^{\prime}\left(k_{r s}^{r} a\right)\right]+k_{s}^{2} a^{2}\left[\mathrm{H}_{0}\left(k_{d s}^{r} a\right) / \mathrm{H}_{0}{ }^{\prime}\left(k_{d s}^{r} a\right)\right]}\right\}
\end{aligned}
$$

2 where $\rho_{f}$ is the density of the contained fluid; the radial wavenumber of the internal fluid, $k_{f s}^{r}$,

3 is related to the fluid wavenumber, $k_{f}$, by $\left(k_{f s}^{r}\right)^{2}=k_{f}^{2}-k_{s}^{2}$ and $k_{f}=\omega / c_{f} ; c_{f}$ is the free-field

4 fluid wavespeed, $c_{f}=\sqrt{B_{f} / \rho_{f}} ; B_{f}$ and $\rho_{f}$ are the bulk modulus and density of the internal

5 fluid; $\mathrm{H}_{0}\left(k_{d s}^{r} r\right)$ and $\mathrm{H}_{0}\left(k_{r s}^{r} r\right)$, represent conical waves radiating outward from the pipe into the

6 surrounding soil; $k_{d s}^{r}$ and $k_{r s}^{r}$, are the radial wavenumbers of the compressional (with the

7 wavenumber $k_{d}$ ) and shear (with the wavenumber $k_{r}$ ) waves respectively, $\left(k_{d s}^{r}\right)^{2}=k_{d}^{2}-k_{s}^{2}$ and

$8 \quad\left(k_{r s}^{r}\right)^{2}=k_{r}^{2}-k_{s}^{2} ; \mathrm{J}_{0}^{\prime}=(\partial / \partial r) \mathrm{J}_{0}() ;$ and $\mathrm{H}_{0}{ }^{\prime}=(\partial / \partial r) \mathrm{H}_{0}()$.

9 For the $s=1$ wave, the relationship between the amplitudes of the axial and radial 10 displacements can be found from Eq. (1) by

$$
U_{1}=\frac{1-\Omega^{2}-F L-S L_{22}}{\mathrm{i} v_{p}\left(k_{1} a\right)+S L_{21}} W_{1}
$$

where the complex wavenumber, $k_{1}$, is obtained by

$$
k_{1}^{2}=k_{f}^{2}\left(1+\frac{\beta}{1-\Omega^{2}+\alpha}\right)
$$


1 direction at the pipe-soil interface, the pressure amplitude, $P_{f 1}$, is found to be related to the

2 radial displacement amplitude of the pipe wall, $W_{1}$, by

3

$$
P_{f 1}=\frac{\omega^{2} \rho_{f}}{k_{f 1}^{r}} \frac{1}{\mathbf{J}_{0}^{\prime}\left(k_{f 1}^{r} a\right)} W_{1}
$$

4 The relationship between the internal pressure and the pipe wall displacements are now set up

5 by combining Eqs. (4) and (6).

\section{$6 \quad 2.2$ Soil vibration}

7

In a cylindrical co-ordinate system, the soil displacements and the stresses may be expressed in terms of the compressional and shear wave potentials, and are given by [7]

$$
\left(\begin{array}{l}
u_{x} \\
u_{r}
\end{array}\right)=\left[\begin{array}{cc}
-\mathrm{i} k_{1} \mathrm{H}_{0}\left(k_{d 1}^{r} r\right) & \left(k_{r 1}^{r}\right)^{2} \mathrm{H}_{0}\left(k_{r 1}^{r} r\right) \\
k_{d 1}^{r} \mathrm{H}_{0}^{\prime}\left(k_{d 1}^{r} r\right) & -\mathrm{i} k_{1} k_{r 1}^{r} \mathrm{H}_{0}^{\prime}\left(k_{r 1}^{r} r\right)
\end{array}\right]\left(\begin{array}{l}
A_{m} \\
B_{m}
\end{array}\right) \mathrm{e}^{\mathrm{i}\left(\omega t-k_{1} x\right)}
$$

10 and

$11\left(\begin{array}{c}\tilde{\sigma}_{r x} \\ \tilde{\sigma}_{r r}\end{array}\right)=\left[\begin{array}{cc}-2 \mathrm{i} \mu_{m} k_{1} k_{d 1}^{r} \mathrm{H}_{0}{ }^{\prime}\left(k_{d 1}^{r} r\right) & -\mu_{m} k_{r 1}^{r}\left(2 k_{1}^{2}-k_{r}^{2}\right) \mathrm{H}_{0}{ }^{\prime}\left(k_{r 1}^{r} r\right) \\ 2 \mu_{m}\left(k_{d 1}^{r}\right)^{2} \mathrm{H}_{0}{ }^{\prime \prime}\left(k_{d 1}^{r} r\right)-\lambda_{m} k_{d}^{2} \mathrm{H}_{0}\left(k_{d 1}^{r} r\right) & -2 \mathrm{i} \mu_{m} k_{1}\left(k_{r 1}^{r}\right)^{2} \mathrm{H}_{0}{ }^{\prime \prime}\left(k_{r 1}^{r} r\right)\end{array}\right]\left(\begin{array}{l}A_{m} \\ B_{m}\end{array}\right) \mathrm{e}^{\mathrm{i}\left(\omega t-k_{1} x\right)}$

12 where $A_{m}$ and $B_{m}$ are the potential coefficients of the compressional and shear waves.

13 Applying the displacement continuity conditions (at $r=a$ ) in both the axial and radial 14 directions, i.e., $u_{x}(a)=u$ and $u_{r}(a)=w$ in Eq. (7) and eliminating the harmonic terms $\mathrm{e}^{\mathrm{i}\left(\omega t-k_{1} x\right)}$, 15 the potential coefficients, $A_{m}$ and $B_{m}$, can be expressed in terms of the amplitudes of the pipe 16 wall displacement, $U_{1}$ and $W_{1}$, by

$$
\left[\begin{array}{l}
A_{m} \\
B_{m}
\end{array}\right]=\frac{\left[\begin{array}{cc}
\mathrm{i} k_{1} \mathrm{H}_{0}{ }^{\prime}\left(k_{r 1}^{r} a\right) & k_{r 1}^{r} \mathrm{H}_{0}\left(k_{r 1}^{r} a\right) \\
\left(k_{d 1}^{r} / k_{r 1}^{r}\right) \mathrm{H}_{0}{ }^{\prime}\left(k_{d 1}^{r} a\right) & \mathrm{i}\left(k_{1} / k_{r 1}^{r}\right) \mathrm{H}_{0}\left(k_{d 1}^{r} a\right)
\end{array}\right]}{k_{r 1}^{r} k_{d 1}^{r} \mathrm{H}_{0}\left(k_{r 1}^{r} a\right) \mathrm{H}_{0}{ }^{\prime}\left(k_{d 1}^{r} a\right)+k_{1}{ }^{2} \mathrm{H}_{0}{ }^{\prime}\left(k_{r 1}^{r} a\right) \mathrm{H}_{0}\left(k_{d 1}^{r} a\right)}\left[\begin{array}{c}
U_{1} \\
W_{1}
\end{array}\right]
$$

It is noted that the relationships between the internal pressure and the pipe wall displacements have been set up by Eqs. (4) and (6). Therefore, combining them with Eq. (9) leads to the potential coefficients, $A_{m}$ and $B_{m}$, which may be expressed in terms of the 
1 amplitudes of the internal pressure $P_{f 1}$ or the pipe wall displacement, $U_{1}$ or $W_{1}$.

2 3. Ground surface motion

Soil vibration has been studied for the fluid-filled pipe buried in an infinite surrounding soil. In this section, further development of the theory is carried out to determine the ground surface motion in response to the radiation of elastic waves from the pipe into the soil.

\subsection{Incident waves in the soil}

Consider the two types of elastic body waves, i.e., the compressional or shear waves that may propagate in the soil medium. Directly over the pipe as illustrated in Fig. 3, for each incident plane wave, both types of elastic waves emanate in a homogeneous and isotropic half space as a result of the reflection at the free surface. In the Cartesian co-ordinates, the horizontal ( $x$ direction) and vertical ( $z$ direction) soil displacements can then be expressed in terms of two wave potentials by [21]

$$
u_{x}=\frac{\partial \phi_{p}}{\partial x}+\frac{\partial \psi_{p}}{\partial z} ; u_{z}=\frac{\partial \phi_{p}}{\partial z}-\frac{\partial \psi_{p}}{\partial x}
$$

where $\phi_{p}$ and $\psi_{p}$ are the compressional and shear potentials respectively, which for plane waves have the form

$$
\phi_{p}=A_{p} \mathrm{e}^{\mathrm{i}\left(\omega t-k_{d 1}^{r} z-k_{1} x\right)} ; \psi_{p}=B_{p} \mathrm{e}^{\mathrm{i}\left(\omega t-k_{r 1}^{r} z-k_{1} x\right)}
$$

with the subscript $p$ denoting plane waves; and $A_{p}$ and $B_{p}$ are potential coefficients. Substituting Eqs. (11) into (10) gives

$$
\left(\begin{array}{l}
u_{x} \\
u_{z}
\end{array}\right)=\left[\begin{array}{cc}
-\mathrm{i} k_{1} \mathrm{e}^{-\mathrm{i} k_{d 1}^{r} z} & -\mathrm{i} k_{r 1}^{r} \mathrm{e}^{-\mathrm{i} k_{r 1}^{r} z} \\
-\mathrm{i} k_{d 1}^{r} \mathrm{e}^{-\mathrm{i} k_{d 1}^{r} z} & \mathrm{i} k_{1} \mathrm{e}^{-\mathrm{i} k_{r 1}^{r} z}
\end{array}\right]\left(\begin{array}{l}
A_{p} \\
B_{p}
\end{array}\right) \mathrm{e}^{\mathrm{i}\left(\omega t-k_{1} x\right)}
$$

Jette and Parker [12] first suggested that the radiated waves travelling over a depth $d$ (calculated from the pipe centre) can be treated as plane waves at the ground surface provided that $\left|k_{d 1}^{r} d\right|>1$ and $\left|k_{r 1}^{r} d\right|>1$ (i.e., for a sufficient large wavenumber-depth product). Here a similar approach is taken to allow for the analytical solutions to the reflection of plane waves 
1 at the free surface. Noting that compressional waves travel faster than shear waves in the soil,

2 if $\left|k_{d 1}^{r} d\right|>1$, then the condition $\left|k_{r 1}^{r} d\right|>1$ is automatically satisfied. To set up the relationship

3 between the two co-ordinate systems, adopting the far-field asymptotic approximations for

4 the Hankel functions in Eq. (7) at $r=d$, and setting the horizontal and vertical soil

5 displacements given by Eq. (12) $(z=d)$ equal to the corresponding soil displacements in the

6 cylindrical co-ordinates, the potential coefficients, $A_{p}$ and $B_{p}$, are found to be related to $A_{m}$

7 and $B_{m}$ by

$$
A_{p}=\sqrt{\frac{2}{\pi k_{d 1}^{r} d}} e^{\mathrm{i} \pi / 4} A_{m} ; B_{p}=\mathrm{i} \sqrt{\frac{2 k_{r 1}^{r}}{\pi d}} e^{\mathrm{i} \pi / 4} B_{m}
$$

\subsection{Reflections at free surface}

When radiated waves are incident upon the ground surface, reflections occur as illustrated in Fig. 3. As stated above, it is assumed that the reflection at the free surface leads to the compressional and shear waves. Correspondingly, the potentials, $\phi_{p}$ and $\psi_{p}$, can be expressed as overall contributions of the incident and reflected wave potentials by

$$
\phi_{p}=\left(A_{p}^{+} \mathrm{e}^{-\mathrm{i} k_{d 1}^{r} z}+A_{p}^{-} \mathrm{e}^{\mathrm{i} k_{d 1}^{r} z}\right) \mathrm{e}^{\mathrm{i}\left(\omega t-k_{1} x\right)} ; \psi_{p}=\left(B_{p}^{+} \mathrm{e}^{-\mathrm{i} k_{r 1}^{r} z}+B_{p}^{-} \mathrm{e}^{\mathrm{i} r_{r 1}^{r} z}\right) \mathrm{e}^{\mathrm{i}\left(\omega t-k_{1} x\right)}
$$

where $A_{p}^{+}$and $B_{p}^{+}$are the incident potential coefficients obtained by Eqs. (13a, b); $A_{p}^{-}$and $B_{p}^{-}$are the unknown reflected potential coefficients. Substituting Eqs. (14) into (10) gives

$$
\left(\begin{array}{l}
u_{x} \\
u_{z}
\end{array}\right)=\left\{\left[\begin{array}{cc}
-\mathrm{i} k_{1} \mathrm{e}^{-\mathrm{i} k_{d 1}^{r} z} & -\mathrm{i} k_{r 1}^{r} \mathrm{e}^{-\mathrm{i} k_{r 1}^{r} z} \\
-\mathrm{i} k_{d 1}^{r} \mathrm{e}^{-\mathrm{i} k_{d 1}^{r} z} & \mathrm{i} k_{1} \mathrm{e}^{-\mathrm{i} k_{r 1}^{r} z}
\end{array}\right]\left[\begin{array}{c}
A_{p}^{+} \\
B_{p}^{+}
\end{array}\right]+\left[\begin{array}{cc}
-\mathrm{i} k_{1} \mathrm{e}^{\mathrm{i} k_{d 1}^{r} z} & \mathrm{i} k_{r 1}^{r} \mathrm{e}^{\mathrm{i} r_{r 1}^{r} z} \\
\mathrm{i} k_{d 1}^{r} \mathrm{e}^{\mathrm{i} k_{d 1}^{r} z} & \mathrm{i} k_{1} \mathrm{e}^{\mathrm{i} k_{r 1}^{r} z}
\end{array}\right]\left[\begin{array}{c}
A_{p}^{-} \\
B_{p}^{-}
\end{array}\right]\right\} \mathrm{e}^{\mathrm{i}\left(\omega t-k_{1} x\right)}
$$

According to Hooke's Law, the stresses are related to the soil displacements by

$$
\tilde{\sigma}_{z x}=\mu_{m}\left(\frac{\partial u_{z}}{\partial x}+\frac{\partial u_{x}}{\partial z}\right) ; \tilde{\sigma}_{z z}=\lambda_{m} \Delta+2 \mu_{m} \frac{\partial u_{z}}{\partial z}
$$




$$
\left(\begin{array}{l}
\sigma_{z x} \\
\sigma_{z z}
\end{array}\right)=-\mu_{m}\left\{\left[\begin{array}{cc}
2 k_{1} k_{d 1}^{r} \mathrm{1}^{-\mathrm{i} k_{d 1}^{r} z} & \left.\left[\left(k_{r 1}^{r}\right)^{2}-k_{1}^{2}\right] \mathrm{e}^{-\mathrm{i} k_{r 1}^{r}}\right] \\
\left(k_{r}^{2}-2 k_{1}^{2}\right) \mathrm{e}^{-\mathrm{i} k_{d 1}^{r} z} & -2 k_{1} k_{r 1}^{r} \mathrm{e}^{-\mathrm{i} k_{r 1}^{r} z}
\end{array}\right]\left[\begin{array}{c}
A_{p}^{+} \\
B_{p}^{+}
\end{array}\right]+\left[\begin{array}{cc}
-2 k_{1} k_{d 1}^{r} \mathrm{e}^{\mathrm{i} k_{d 1}^{r} z} & {\left[\left(k_{r 1}^{r}\right)^{2}-k_{1}^{2}\right] \mathrm{e}^{\mathrm{i} k_{r 1}^{r} z}} \\
\left(k_{r}^{2}-2 k_{1}^{2}\right) \mathrm{e}^{\mathrm{i} k_{d 1}^{r} z} & 2 k_{1} k_{r 1}^{r} \mathrm{e}^{\mathrm{i} k_{r 1}^{r} z}
\end{array}\right]\left[\begin{array}{l}
A_{p}^{-} \\
B_{p}^{-}
\end{array}\right]\right\} \mathrm{e}^{\mathrm{i}\left(\omega t-k_{1} x\right)}
$$

2

3

$$
\left(\begin{array}{c}
A_{p}^{-} \\
B_{p}^{-}
\end{array}\right)=\left[\begin{array}{cc}
C_{11} \mathrm{e}^{-2 \mathrm{i} k_{d 1}^{r} d} & C_{12} \mathrm{e}^{-\mathrm{i}\left(k_{d 1}^{r}+k_{r 1}^{r}\right) d} \\
C_{21} \mathrm{e}^{-\mathrm{i}\left(k_{d 1}^{r}+k_{r 1}^{r}\right) d} & C_{11} \mathrm{e}^{-2 \mathrm{i} k_{r 1}^{r} d}
\end{array}\right]\left(\begin{array}{c}
A_{p}^{+} \\
B_{p}^{+}
\end{array}\right)
$$

4 where

$$
C_{11}=\frac{4 k_{1}^{2} k_{d 1}^{r} k_{r 1}^{r}-\left[\left(k_{r 1}^{r}\right)^{2}-k_{1}^{2}\right]^{2}}{4 k_{1}^{2} k_{d 1}^{r} k_{r 1}^{r}+\left[\left(k_{r 1}^{r}\right)^{2}-k_{1}^{2}\right]^{2}}
$$$$
C_{12}=\frac{4 k_{1} k_{r 1}^{r}\left[\left(k_{r 1}^{r}\right)^{2}-k_{1}^{2}\right]}{4 k_{1}^{2} k_{d 1}^{r} k_{r 1}^{r}+\left[\left(k_{r 1}^{r}\right)^{2}-k_{1}^{2}\right]^{2}}
$$

$5 \quad C_{21}=-\frac{4 k_{1} k_{d 1}^{r}\left[\left(k_{r 1}^{r}\right)^{2}-k_{1}^{2}\right]}{4 k_{1}^{2} k_{d 1}^{r} k_{r 1}^{r}+\left[\left(k_{r 1}^{r}\right)^{2}-k_{1}^{2}\right]^{2}}$.

Substituting Eqs. (18) into (15) results in the soil displacements at the ground surface in 7 terms of the potential coefficients of incident waves by

$$
\left(\begin{array}{l}
u_{x} \\
u_{z}
\end{array}\right)=\left(\mathbf{U}_{d} A_{p}^{+} \mathrm{e}^{-\mathrm{i} k_{d 1}^{r} d}+\mathbf{U}_{r} B_{p}^{+} \mathrm{e}^{-\mathrm{i} k_{r 1}^{r} d}\right) \mathrm{e}^{\mathrm{i}\left(\omega t-k_{1} x\right)}
$$

$9 \quad$ where the amplitude vectors $\mathbf{U}_{d}$ and $\mathbf{U}_{r}$ are given by

$$
\mathbf{U}_{d}=-\mathrm{i}\left[\begin{array}{l}
k_{1}\left(1+C_{11}\right)-k_{r 1}^{r} C_{21} \\
k_{d 1}^{r}\left(1-C_{11}\right)-k_{1} C_{21}
\end{array}\right] ; \mathbf{U}_{r}=-\mathrm{i}\left[\begin{array}{l}
k_{r 1}^{r}\left(1-C_{11}\right)+k_{1} C_{12} \\
-k_{1}\left(1+C_{11}\right)-k_{d 1}^{r} C_{12}
\end{array}\right]
$$

It is noted that the free wavenumbers, $k_{d}$ and $k_{r}$, can be vectorially decomposed into $x$ trace (axial) component, $k_{1}$, and $z$ trace (radial) components, $k_{d 1}^{r}$ and $k_{r 1}^{r}$, respectively, as given in Section 2.1. This explains that in practice, the $s=1$ wave can be effectively observed at the trace velocity of $c_{1}$ when making the ground surface measurements. As can be seen 16 overall contributions made by both plane waves with the trace components, $k_{d 1}^{r}$ and $k_{r 1}^{r}$, 17 radiating outward from the pipe over a propagation distance $d$. Consequently, the nature of 18 the ground surface vibration induced by the propagating $s=1$ wave is predominantly affected by the radiation condition along with the burial depth. Furthermore, at the free surface, the 
1 incident wave potential coefficients, $A_{p}^{+}$and $B_{p}^{+}$, can be approximated by Eqs. (13a, b). By

2 eliminating the harmonic terms $\mathrm{e}^{\mathrm{i}\left(\omega t-k_{1} x\right)}$ on both sides in Eq. (19), the amplitudes of the soil

3 displacements, $U_{x}$ and $U_{z}$, can be expressed in terms of the potential coefficients, $A_{m}$ and $B_{m}$, 4 as

5

$$
\left[\begin{array}{c}
U_{x} \\
U_{z}
\end{array}\right]=\sqrt{\frac{2}{\pi k_{d 1}^{r} d}} e^{\mathrm{i} \pi / 4} \mathbf{U}_{d} A_{m} \mathrm{e}^{-\mathrm{i} k_{d 1}^{r} d}+\mathrm{i} \sqrt{\frac{2 k_{r 1}^{r}}{\pi d}} \mathbf{U}_{r} B_{m} \mathrm{e}^{-\mathrm{i} k_{r 1}^{r} d}
$$

The first and second terms on the right side of Eq. (21) indicate the contributions of the $z$

7 trace components made by the compressional and shear waves respectively. Here, the 8 potential coefficients, $A_{m}$ and $B_{m}$, are given by Eq. (9).

\section{$9 \quad 3.3$ Elastic waves in representative soils}

The vibrational behaviour of the ground surface results from the combination of two types of elastic waves which may radiate in the soil, with the total contribution satisfying the boundary conditions at the free surface. Here, two soil types may be distinguished: those for which both the shear and compressional wavespeeds are both less than the wavespeed in the pipe; and those for which the compressional wavespeed is greater than that in the pipe. Typically, sandy soils fall into the first category with and clay/chalky soils falling into the second, as shown in Table 1.

As might be expected, the elastic properties of the soil are of great importance in influencing the radiation induced by the $s=1$ wave that propagates in a fluid-filled pipe. In the following, it should be noted that the real part of the wavenumber is related to the phase velocity of the propagating wave. Recent work by the present authors [7] has shown that the $s=1$ wave, will leak shear waves for typical values of the shear velocity in the soil (when $c_{1}>c_{r}$ or $\left.\operatorname{Re}\left(k_{1}^{2}\right)<\operatorname{Re}\left(k_{r}^{2}\right)\right)$, while may or may not leak compressional waves into the soil.

23 For example, the compressional wave will radiate in loose, sandy soils (where, in general, $c_{1}>c_{d}$ or $\operatorname{Re}\left(k_{1}^{2}\right)<\operatorname{Re}\left(k_{d}^{2}\right)$ ), but will be less likely to radiate in clay soils (where, typically 
$1 \quad c_{1}<c_{d}$ or $\left.\operatorname{Re}\left(k_{1}^{2}\right)>\operatorname{Re}\left(k_{d}^{2}\right)\right)$. We therefore consider the following radiation conditions:

(1) For $c_{1}>c_{d}$, the $s=1$ wave leaks both compressional and shear waves. In this case, it can be seen from Eq. (21) that the overall soil displacements in both the horizontal and vertical directions at the ground surface consist of a superposition of the responses due to both wave types. When both the compressional and shear waves radiate, the interference effect of the waves will result in a complex oscillation at the ground surface due to their different phase velocities.

(2) For $c_{1}<c_{d}$, only the shear wave radiates in the soil. Setting $A_{p}^{+}=0$, it is clear that the contribution of the radiated compressional wave vanishes in Eq. (21). This lead to

$$
\left[\begin{array}{l}
U_{x} \\
U_{z}
\end{array}\right]=\mathrm{i} \sqrt{\frac{2 k_{r 1}^{r}}{\pi d}} \mathbf{U}_{r} B_{m} \mathrm{e}^{-\mathrm{i} k_{r 1}^{r} d}
$$

The effect of radiated elastic waves on the ground surface displacements is examined numerically in two representative soils in the next section.

\section{Numerical results and discussions}

This section presents some numerical results of the ground surface displacements relative to the pipe wall displacements induced by the $s=1$ wave motion in a buried water-filled PVC pipe (of diameter $160 \mathrm{~mm}$ and thickness $10 \mathrm{~mm}$ ). Two representative soils are considered: a typical sandy soil (A) and a typical clay soil (B), which provide consistency with the previous study carried out in [7]. Losses within the surrounding medium are included (with a loss factor of 0.1) by adopting complex elastic moduli for the soil. The material properties of the pipe, soil and fluid are shown in Table 2. The frequency range of interest is up to $1 \mathrm{kHz}$, since signals are heavily attenuated at higher frequencies in plastic pipes.

\subsection{Phase velocity of the $s=1$ wave}

The phase velocity of the $s=1$ wave is plotted in Figs. 4(a) and (b) for sandy soil (A) and clay soil (B) respectively. For both soil types, the propagating $s=1$ wave is slightly dispersive. 
1 This is caused by the added effect of soil loading on the pipe wall, more details of which can

2 be found in [7]. It can be seen from Fig. 4(a) that the calculated phase velocity of the $s=1$

3 wave for the sandy soil is greater than both the compressional and shear velocities in the soil.

4 This suggests that both elastic waves will radiate in the sandy soil considered. For clay soil

5 (B), however, the phase velocity of the $s=1$ wave lies between the shear and compressional

6 velocities, as shown in Fig. 4(b). In this case, the $s=1$ wave will only leak the shear wave.

$7 \quad 4.2$ Ground surface displacements

In this section, the main factors that affect the vibrational behaviour at the ground surface are studied, including the soil type and the burial depth (calculated above the pipe). In the numerical analysis, the ground surface displacements, $\tilde{U}_{x}$ and $\tilde{U}_{z}$, are evaluated in terms of

11 the non-dimensional amplification factors relative to the radial displacement of the pipe wall,

12 i.e., $\tilde{U}_{x}=U_{x} / W_{1}, \tilde{U}_{z}=U_{z} / W_{1}$. Substituting Eqs (4), (9) into (21) gives

$$
\tilde{U}_{x}=\frac{\left\{\begin{array}{c}
{\left[k_{1}\left(1+C_{11}\right)-k_{r 1}^{r} C_{21}\right] \sqrt{\frac{2}{\pi k_{d 1}^{r} d}} e^{\mathrm{i} \pi / 4}\left[k_{1} \mathrm{H}_{0}{ }^{\prime}\left(k_{r 1}^{r} a\right) \frac{1-\Omega^{2}-F L-S L_{22}}{\mathrm{i} v_{p}\left(k_{1} a\right)+S L_{21}}-\mathrm{i} k_{r 1}^{r} \mathrm{H}_{0}\left(k_{r 1}^{r} a\right)\right] \mathrm{e}^{-\mathrm{i} k_{d 1}^{r} d}} \\
+\left[k_{r 1}^{r}\left(1-C_{11}\right)+k_{1} C_{12}\right] \sqrt{\frac{2}{\pi k_{r 1}^{r} d}}\left[k_{d 1}^{r} \mathrm{H}_{0}{ }^{\prime}\left(k_{d 1}^{r} a\right) \frac{1-\Omega^{2}-F L-S L_{22}}{\mathrm{i} v_{p}\left(k_{1} a\right)+S L_{21}}+\mathrm{i}_{1} \mathrm{H}_{0}\left(k_{d 1}^{r} a\right)\right] \mathrm{e}^{-\mathrm{i} k_{r 1}^{r} d}
\end{array}\right\}}{k_{r 1}^{r} k_{d 1}^{r} \mathrm{H}_{0}\left(k_{r 1}^{r} a\right) \mathrm{H}_{0}{ }^{\prime}\left(k_{d 1}^{r} a\right)+k_{1}{ }^{2} \mathrm{H}_{0}{ }^{\prime}\left(k_{r 1}^{r} a\right) \mathrm{H}_{0}\left(k_{d 1}^{r} a\right)}
$$

$$
\tilde{U}_{z}=\frac{\left\{\begin{array}{c}
{\left[k_{d 1}^{r}\left(1-C_{11}\right)-k_{1} C_{21}\right] \sqrt{\frac{2}{\pi k_{d 1}^{r} d}} e^{\mathrm{i} \pi / 4}\left[k_{1} \mathrm{H}_{0}^{\prime}\left(k_{r 1}^{r} a\right) \frac{1-\Omega^{2}-F L-S L_{22}}{\mathrm{i} v_{p}\left(k_{1} a\right)+S L_{21}}-\mathrm{i} k_{r 1}^{r} \mathrm{H}_{0}\left(k_{r 1}^{r} a\right)\right] \mathrm{e}^{-\mathrm{i} k_{d 1}^{r} d}} \\
+\left[-k_{1}\left(1+C_{11}\right)-k_{d 1}^{r} C_{12}\right] \sqrt{\frac{2}{\pi k_{r 1}^{r} d}}\left[k_{d 1}^{r} \mathrm{H}_{0}^{\prime}\left(k_{d 1}^{r} a\right) \frac{1-\Omega^{2}-F L-S L_{22}}{\mathrm{i} v_{p}\left(k_{1} a\right)+S L_{21}}+\mathrm{i} k_{1} \mathrm{H}_{0}\left(k_{d 1}^{r} a\right)\right] \mathrm{e}^{-\mathrm{i} r_{r 1}^{r} d}
\end{array}\right\}}{k_{r 1}^{r} k_{d 1}^{r} \mathrm{H}_{0}\left(k_{r 1}^{r} a\right) \mathrm{H}_{0}^{\prime}\left(k_{d 1}^{r} a\right)+k_{1}^{2} \mathrm{H}_{0}^{\prime}\left(k_{r 1}^{r} a\right) \mathrm{H}_{0}\left(k_{d 1}^{r} a\right)}
$$

with the first and second terms representing the contributions of the compressional and shear waves respectively.

\subsubsection{Sandy soil}

For the water-filled PVC pipe buried at a depth of $1 \mathrm{~m}$, the theory presented here can be 
1 adopted to predict the displacements above $33 \mathrm{~Hz}$ for $\left|k_{d}^{r} d\right|>1$. As discussed in Section 4.1,

2 both compressional and shear waves radiate in the sandy soil. Therefore, the interfering effect

3 of the elastic waves is anticipated in the plots of the ground surface displacement. For the 4 magnitude plots of both the horizontal and vertical amplification factors, as can be seen from 5 Fig. 5(a) and Fig. 6(a), the shear wave contributions are much greater than the compressional 6 wave contributions at lower frequencies; they decrease rapidly with increasing frequency 7 whereas the contributions due to the compressional wave increase. As a result, it is observed 8 that both the overall horizontal and vertical magnitudes fluctuate in the frequency range of 9 interest. Fig. 5(a) shows that for the horizontal displacement, the fluctuations in the 10 magnitude lead to a series of local maxima and minima separated by about $200 \mathrm{~Hz}$. In 11 particular, the magnitude reaches the minimum value (approximately zero) at $590 \mathrm{~Hz}$. For the 12 vertical displacement, it can be found from Fig. 6(a) that the magnitude fluctuates with a 13 separation of roughly $160 \mathrm{~Hz}$ at lower frequencies below $600 \mathrm{~Hz}$ and the oscillatory 14 behaviour becomes less obvious at higher frequencies.

To demonstrate the interfering effect of two waves on the phase of the ground surface displacements, both the wrapped and unwrapped phase are plotted in Figs. 5(b) and (c) for the horizontal amplification factors, and in Figs. 6(b) and (c) for the vertical amplification factors respectively. For the horizontal displacement, as can be seen from Fig. 5(b), the contribution of the shear wave dominates below $400 \mathrm{~Hz}$. Above $400 \mathrm{~Hz}$, the response deviates from the radial shear wave component since the radiated compressional wave contributes significantly. In particular at $590 \mathrm{~Hz}$, an abrupt phase change occurs as marked by the red arrow in the phase plot. The occurrence of the phase changes are easily explained by 23 re-expressing the displacements given by Eq. (23), in a simplified form of $\tilde{U}_{x}=g_{1} \mathrm{e}^{\mathrm{i} \xi_{1}}+g_{2} \mathrm{e}^{\mathrm{i} \xi_{1}}$. 24 Here $g_{1}$ and $g_{2}$ are the magnitudes for two elastic waves, and $\zeta_{1}$ and $\zeta_{2}$ are the corresponding 25 phases. When the overall magnitude of $\tilde{U}_{x}$ approaches a minimum of zero, the lag of one 
1 displacement term with respect to another must approach $\pm \pi$. This leads to the abrupt phase

2 change at the frequencies coincident with magnitude minima. Further check of the

3 unwrapped phase plotted in Fig. 5(c) shows that the phase varies approximately linearly with

4 frequency at lower frequencies, with the radiated shear wave being dominant. Again, at $5590 \mathrm{~Hz}$, an abrupt phase change occurs; above this frequency, it shifts towards the phase of

6 the radial compressional wavenumber component, as the contribution of the compressional

7 wave increases. In contrast, for the vertical displacement, the unwrapped phase is mainly

8 governed by the radial compressional wavenumber component at higher frequencies above

$9140 \mathrm{~Hz}$. Similar to the horizontal displacement, a phase change occurs at around $80 \mathrm{~Hz}$ where

10 the magnitude approaches a local minimum. Clearly both the horizontal and vertical 11 displacements of the ground surface are affected by the interfering effect of the elastic waves 12 in the soil.

\section{$13 \quad 4.2 .2 \quad$ Clay soil}

14 For a burial depth of $1 \mathrm{~m}$, the shear wavenumber satisfies $\left|k_{r}^{r} d\right|>1$ above $51 \mathrm{~Hz}$. Figs. 7

and 8 plot the horizontal and vertical amplification factors for clay soil (B). A similar trend can be seen from the magnitude results plotted in Figs 7(a) and 8(a). Compared with the sandy soil plotted in Figs. 5(a) and 6(a), the magnitude increases with frequency in particular above $800 \mathrm{~Hz}$ for the clay soil (being greater than 1). It can be seen from the magnitude plots that there is no evident interfering behaviour of the elastic waves. Figs. 7(b) and 8(b) plot the phase of the horizontal and vertical amplification factors respectively. When only the shear wave radiates in the soil, as anticipated, no abrupt phase changes are observed. In addition, it can be seen from Eq. (22) that the phase is directly related to the radial shear wavenumber. This may offer an alternative way to retrieve the $s=1$ phase velocity from ground surface measurements, provided the shear wavespeed in the soil is known, along with the pipe depth. Alternatively, it offers a way to estimate the pipe depth provided the $s=1$ phase velocity can 
1 be estimated from the spatial variation of phase above the pipe.

$2 \quad 4.2 .3$ Effect of the burial depth

3 In sandy soil (A) where both elastic waves radiate, the amplification factors are plotted in 4 Figs. (9) and (10) for different burial depths. Above $60 \mathrm{~Hz}, 33 \mathrm{~Hz}$ and $24 \mathrm{~Hz}$, the 5 wavenumber-depth product satisfies $\left|k_{d}^{r} d\right|>1$ for the depth of $0.5 \mathrm{~m}, 1 \mathrm{~m}$ and $1.5 \mathrm{~m}$

6 respectively. For both horizontal and vertical displacements, the magnitude and phase plotted

7 in Figs. (9a-c) and (10a-c) illustrate very similar low-frequency vibrational behaviour for 8 different burial depths. As can be seen from the magnitude plots, the magnitude level 9 decreases slightly due to the increasing attenuation with burial depths. The interfering effect 10 of elastic waves becomes more evident with a narrower frequency separation for the deep 11 depth with the penalty in the reduction of the magnitude level. Therefore, examination of the 12 fluctuations in the magnitude of the ground surface response may provide the additional 13 information required to estimate the burial depth.

14 Again, it can be seen from Figs. 9(d-f) and 10(d-f) that distinct phase changes occur in the 15 plots of the horizontal amplification factor at frequencies when the corresponding magnitudes 16 approach local minima.

\section{Experimental measurements}

Here some experimental measurements are presented to support the theoretical results shown in the previous sections. The measurements were carried out on a dedicated pipe rig, the construction of which has been detailed previously [22]. However, for clarity, a description of the main features is also included here.

\subsection{Experimental rig}

The rig consists of an approximately $18 \mathrm{~m}$ length of MDPE (medium density polyethylene) water pipe (180 $\mathrm{mm}$ OD, $11 \mathrm{~mm}$ wall thickness) buried at a depth of approximately $1 \mathrm{~m}$ (the standard burial depth for mains water pipes in the UK). Table 3 gives 
1 the key geometrical and material properties of the pipe. A right-angled bend brings the pipe

2 up to the ground surface at one end, and a man-hole provides access at the other. This allows

3 direct access to the pipe at either end of the rig; flanged pipe ends enable each end to be

4 sealed with a blanking plate or not as required. The rig was designed with the excitation and

5 propagation of the $n=0, s=1$ wave in mind. The pipe is instrumented with a number of PVDF

6 (polyvinylidene fluoride) ring sensors [4] which allow this particular wave to be monitored

7 [22]. In particular the ring sensors measure the radial pipe wall displacement needed for the

8 present study.

9 The general layout of the rig is shown in Fig. 11, along with the coordinate axes used for 10 the measurements. The soil in which the pipe was buried was a mixture of gravel, sand and 11 clay. Preliminary measurements have been undertaken previously on the rig, in order to 12 determine the best way to excite this wave-type [22]. Of the methods tested, it was found that

13 it could be most effectively excited with an inertial shaker mounted on a plastic end-plate 14 bolted to the flanged end of the pipe as it comes up to the ground surface; an additional 15 advantage of this particular arrangement is that no direct contact with the water inside the 16 pipe is required and the pipe wall only need be excited. This arrangement is shown in Fig. 12.

\subsection{Pipe wall and ground vibration measurements}

The shaker was excited with a swept sine input from $30 \mathrm{~Hz}$ to $400 \mathrm{~Hz}$ over a period of approximately 4 minutes, with the force applied by the shaker being measured simultaneously, at a sampling rate of $1000 \mathrm{~Hz}$. The pipe wall displacement resulting from excitation of the pipe was measured at two locations along the pipe, using the PVDF wire installed on the rig, $2 \mathrm{~m}$ and $3 \mathrm{~m}$ axially from the source end of the pipe. Two measurements were taken to enable estimates of the wavespeed in the pipe to be made (it had been established previously [12, 22] that the magnitude of the reflections from the far end of the pipe were sufficiently low to not require a third measurement location). 
Vertical and horizontal (in line with the pipe) ground vibration velocity measurements were made using I-O SM24 geophones positioned on the ground directly above the second PVDF rig sensor $3 \mathrm{~m}$ from the excitation location. The natural frequency of the geophones was $10 \mathrm{~Hz}$, so they give a flat response at frequencies above $30 \mathrm{~Hz}$. Frequency response functions were calculated relating both the radial pipe wall displacements and the ground surface velocities to the input force measured at the shaker.

\subsection{Experimental results}

Figs. 14(a) and (b) depict the magnitude and phase of the pipe wall and ground surface displacements relative to the input force. Below $30 \mathrm{~Hz}$, the signals are dominated by noise as expected. Spikes in the plot at odd harmonics of $50 \mathrm{~Hz}$ are related to mains interference. Fig. 14(a) shows that all four measurements exhibit similar trends, with an overall decrease in the magnitudes with increasing frequency. This is as expected given the large attenuation at higher frequencies for both waves in the pipe and in the soil. The geophone data becomes increasingly noisy at high frequencies. Moreover, all the measurements exhibit oscillatory behaviour with clear peaks and troughs in the response, suggesting some interference mechanism in play, particularly for the ground surface responses between $150 \mathrm{~Hz}$ and $200 \mathrm{~Hz}$. This is as expected given that both the soil shear wave and compressional wavespeeds (see Table 4) are less than the wavepeed in the pipe. Examining the phase response in Fig. 14(b), above $30 \mathrm{~Hz}$, the phase of all the measurements exhibit an approximately linear variation with frequency (as would be expected for the near-non-dispersive wave propagating in the pipe). Superimposed on this linear variation, and coincident with the troughs in Fig. 14(a), are phase jumps in the ground surface response, akin to those described earlier in the present paper.

\subsection{Wavespeed estimation and soil properties}

In order to select the most appropriate soil properties for comparison of the experimental 
1 measurements with the theory presented, we have been guided by a combination of the

2 measurements themselves, previous seismic surveys made on the same site [23] and

3 knowledge of the overall soil composition. These soil parameters are shown in Table 4.

4 Moreover, previous work has shown [24] that for a buried pipe at low frequencies, the

5 effect of the soil loading on the $n=0, s=1$ pipe wavespeed is controlled largely by the value of

6 the shear modulus of the soil. Fig. 15 shows the real part of the predicted $s=1$ wavenumber

7 using the parameters shown in Table 4 alongside the wavenumber calculated from

8 measurements made on the pipe wall, confirming the appropriateness of the choices made in

9 the frequency range of interest.

\section{$10 \quad 5.5$ Comparison with theoretical predictions}

11 Figs. 16 and 17 show the magnitude and phase of the horizontal and vertical amplification 12 factors respectively as predicted by theory and as obtained from the experiments. Two 13 predictions are shown for each case: one with a zero loss factor for the soil; and one with a $14 \quad$ loss factor of 0.1

15 Considering first of all the magnitude plots, there are clearly some considerable 16 differences between the measurements and predictions for both the vertical and horizontal 17 responses, particularly at the lower frequencies, where the measured ground surface response 18 is significantly larger than the predicted response. However, above $200 \mathrm{~Hz}$, the predictions 19 and measurements are similar, with the measured response lying between the two predictions 20 for the horizontal amplification factor and matching more closely the lossy case for the 21 vertical amplification factor. However, there are a greater number of dips in the measured response compared with the predicted response, and in general they do not align. A number of 23 possible reasons for the discrepancies between the predictions and the measured data are now 24 proffered.

- It is known that the ground on which the measurements were made is not homogeneous 
with depth, but distinctly layered. Previous investigations revealed that, below approximately $1 \mathrm{~m}$ (the depth of the pipe) a significantly stiffer soil layer exists [23] with a shear modulus of around $2000 \mathrm{GN} / \mathrm{m}^{2}$, extending several metres down. The existence of this layer is likely to affect the ground surface response presented in this paper in two main ways. Firstly, a significant amount of energy radiating from the pipe will be reflected from the interface between the two soil layers, resulting in a much greater response at the ground surface than might be expected in non-layered soil. Due to the attenuation in the soil, this is likely to be more pronounced at lower frequencies, as is indeed the case here. Moreover, the existence of the stiff layer means that, at the ground surface there will be more than two waves interfering (the directly radiated compressional and shear waves). In addition, there may be multiple waves (both compressional and shear), reflected and re-reflected from the stiff layer boundary. This will result in additional interference dips in the response, as is indeed seen in the measured data.

- It is also arguable that the soil cannot be considered homogeneous at all. As stated earlier, Biot theory $[19,20]$ takes soil porosity into account and allows for the possibility of wave types in addition to those considered here. The effects of these waves (particularly in terms of wave interactions) might account for some of the discrepancies between the measured data and the predictions.

- The presented theory assumed a far-field type response at the ground surface. Whilst for relatively low frequencies $\left(<30 \mathrm{~Hz}\right.$ ), the conditions, $\left|k_{d}^{r} d\right|>1$ and $\left|k_{r}^{r} d\right|>1$ are satisfied, it must be borne in mind that the expressions derived are nevertheless approximations whose approximate nature lessens with increasing frequency. The nature of the approximations may mitigate some of the observed differences when compared with the measured response at low frequencies. 
The remaining assumption to be challenged is the supposition that the effects of the soil on the pipe and the effects of the waves propagating in the pipe on the soil can be considered independently, i.e. that in calculating the dispersion relationships for the pipe waves, the effects of the free surface can be ignored. However, this assumption is unlikely to be the cause of discrepancies between the predictions and the measured ground vibration data as the pipe wavenumber measurements and predictions show good agreement. By examining the measured phase, however, some additional insights may be gained. The figures show that, in general, the trends of the phase are reasonably well reproduced. In particular, the unwrapped phase gradients of the measurements reveal a good match with the predictions. As has been noted previously, the overall phase behaviour of the horizontal amplification factor is controlled by the radial shear wavenumber component, with the phase of the vertical amplification factor being controlled by the radial compressional wavenumber component. These are also shown in the figures, where it is seen that the gradients match well with the overall gradients in the measured data. This provides further evidence that the soil properties used for the comparison, in Table 4, are indeed representative of the soil on the measurement test site. What is also particularly interesting to note is that, for the vertical amplification factor plotted in Fig. 17, the gradient of the predicted unwrapped phase is increased relative to the radial compressional wavenumber between $50 \mathrm{~Hz}$ and $150 \mathrm{~Hz}$; this trend is mirrored in the measured response, although here it is significantly more pronounced.

\section{Conclusions}

Based on a model of axisymmetric wave propagation established in recent work, the ground surface displacements due to waves radiating from a buried fluid-filled pipe have been investigated. An analytical method is adopted to derive general expressions for the horizontal and vertical displacements at the ground surface. Theoretical predictions of the ground surface vibration in two representative soils show the following: 
- In sandy soil, the $s=1$ wave leaks both compressional and shear waves. As a result, the interfering effect can lead to the abrupt phase changes that occur at frequencies coincident with magnitude minima in the ground surface displacements. In addition, particularly at low frequencies, the horizontal displacement is dominated by the radiated shear wave whereas the vertical displacement is controlled by the radiated compressional wave.

- In the clay soil, the $s=1$ wave leaks shear waves only. Therefore, as anticipated, no interfering effect can be observed in either the horizontal or vertical amplification factors.

The predictions are supported by measurements made on a dedicated pipe rig. Here, although there are some differences between the measured and predicted response magnitudes at the ground surface, the general trends of the response have been replicated. In particular, the phase response shows good agreement and the interference phenomena occur in both predictions and measurements. Where differences are observed, possible reasons for the discrepancies have been offered. The theoretical model presented in this paper underpins an acoustic technique for locating buried pipes described in an earlier study [11]. Moreover, it offers additional insights into how the technique might be improved such that additional information about the pipe, such as its depth, can be gleaned.

\section{Acknowledgements}

The authors greatly acknowledge the CAS Hundred Talents Programme and the support provided by the EPSRC (under grant EP/K021699/1). Data supporting this study are openly available from the University of Southampton repository at http://dx.doi.org/10.5258/SOTON/405402.

\section{Appendix. Properties of Bessel and Hankel functions}

In this appendix, the properties of Bessel and Hankel functions used in the paper are given as follows [25]: 
- For small argument $x \rightarrow 0, \mathrm{~J}_{0}(x) / \mathrm{J}_{0}{ }^{\prime}(x) \approx-2 / x$;

2

3

6 [1] <www.dxgx.org>.

7 [2] <www.mappingtheunderworld.ac.uk>.

8 [3] C.R. Fuller, F.J. Fahy, Characteristics of wave-propagation and energy-distributions in

- For argument $x, \mathrm{H}_{1}^{\prime}(x)=\mathrm{H}_{0}(x)-\mathrm{H}_{1}(x) / x$ and $\mathrm{H}_{0}{ }^{\prime}(x)=-\mathrm{H}_{1}(x)$;

- For large argument $|x|>1$, the Hankel functions can be approximated by the asymptotic expansions, $\mathrm{H}_{n}(x) \approx \sqrt{2 / \pi x} e^{-\mathrm{i}(x-n \pi / 2-\pi / 4)}$ for an integer $n$.

\section{References} cylindrical elastic shells filled with fluid, Journal of Sound and Vibration 81 (1982) $501-518$.

[4] R.J. Pinnington, A.R. Briscoe, Externally applied sensor for axisymmetrical waves in a fluid-filled pipe, Journal of Sound and Vibration 173 (1994) 503-516)

[5] R. Long, P. Cawley, M. Lowe, Acoustic wave propagation in buried iron water pipes, Proceedings of the Royal Society A 459 (2003) 2749-2770.

[6] R. Long, M. Lowe, P. Cawley, Attenuation characteristics of the fundamental modes that propagate in buried iron water pipes, Ultrasonics 41 (2003) 509-519.

[7] Y. Gao, F. Sui, J.M. Muggleton, J. Yang, Simplified dispersion relationships for fluiddominated axisymmetric wave motion in buried fluid-filled pipes, Journal of Sound and Vibration 375 (2016) 386-402.

[8] J.M. Muggleton, M. Kalkowski, Y. Gao, E. Rustighi, A theoretical study of torsional waves in buried pipes for pipeline condition assessment and monitoring, Journal of Sound and Vibration 374 (2016) 155-171. 
1 [9] Y. Gao, Y. Liu, J.M. Muggleton, Axisymmetric fluid-dominated wave in fluid-filled plastic pipes: loading effects of surrounding elastic medium, Applied Acoustics 116 (2017) 43-49.

[10] G.F. Kuhn, C.L. Morfey, Transmission of low-frequency internal sound through pipe walls, Journal of Sound and Vibration 47 (1976) 147-161.

[11] J.M. Muggleton, M.J. Brennan, Y. Gao, Determining the location of buried plastic water pipes from measurements of ground surface vibration, Journal of Applied Geophysics 75 (2011) 54-61.

[12] A.N. Jette, J.G. Parker, Surface displacement accompanying the propagation of acoustic waves within an underground pipe, Journal of Sound and Vibration 69 (2) (1980) 265-274.

[13] T. Akiyoshi, K. Fuchida, Soil-pipeline interaction through a frictional interface during earthquakes, Soil Dynamics and Earthquake Engineering 3(1) (1984) 27-34.

[14] J.P. Dwivedi, V.P. Singh, P.C. Upadhyay, Effect of fluid presence on the dynamic response of imperfectly bonded buried orthotropic cylindrical shells. Journal of Sound and Vibration 139 (1990) 99-110.

[15] A.A. Stamos, D.E. Beskos, 3D seismic response analysis of long lined tunnels in halfspace, Soil Dynamics and Earthquake Engineering 15 (1996) 111-118.

[16] A. Tadeu, P.A. Mendes, J. Antonio, 3D elastic wave propagation modeling in the presence of 2D fluid-filled thin inclusion, Soil Dynamics and Earthquake Engineering 30 (2006) 176-193.

[17] Y. Nassira, H.Rajaie, R.T.Faal, Three dimensional vibration analysis of a buried pipeline with slip conditions, Soil Dynamics and Earthquake Engineering 31 (2011) $1629-1639$.

[18] K.F. Graff, Wave Motion in Elastic Solids, Dover Publications Inc., New York, 1991. 
[19] M.A. Biot, General theory of three-dimensional consolidation, Journal of Applied

2

3

4 5 Physics 12(2) (1941) 155-164.

[20] M.A. Biot, Theory of propagation of elastic waves in a fluid-saturated porous solid. 1. Low-frequency range, Journal of the Acoustical Society of America 28(2) (1956) 168178.

[21] W.M. Ewing, W.S. Jardetzky, F. Press, Elastic waves in layered media, McGraw-Hill, New York, 1957.

[22] J. M. Muggleton, M. J. Brennan, The design and instrumentation of an experimental rig to investigate acoustic methods for the detection and location of underground piping systems, Applied Acoustics 69 (2008) 1101-1107.

[23] M Iodice, Road and soil dynamic charaterization from surface measurements, $\mathrm{PhD}$ Thesis, University of Southampton 2017.

[24] J.M. Muggleton, J. Yan, Wavenumber prediction and measurement for buried fluidfilled pipes: inclusion of shear coupling at a lubricated pipe/soil interface, Journal of Sound and Vibration 332(5) (2013) 1216-1230.

[25] M. Abramowitz, I.A. Stegun, Handbook of Mathematical Functions, Dover publications, New York, 1970. 
2

\section{List of figure captions}

2 Fig. 1. Plot of the unwrapped phase measured by geophones showing abrupt phase changes

3 between straight line sections [11].

4 Fig. 2. The cylindrical co-ordinate system for a fluid-filled pipe surrounded by an infinite 5 elastic medium.

6 Fig. 3. Illustration of reflection of the radiated elastic waves induced by the fluid-borne wave 7 motion in the Cartesian co-ordinate $(x, y, z)$ system. $c_{1}, c_{d}$ and $c_{r}$ denote the phase velocity of 8 the $s=1$ wave, compressional and shear velocities in the soil respectively.

9 Fig. 4. Phase velocities of the $s=1$ wave for a PVC water pipe buried in: (a) sandy soil A and 10 (b) clay soil (B) in comparison with the compressional $\left(c_{d}\right)$ and shear velocities $\left(c_{r}\right)$ in the 11 surrounding soil.

12 Fig. 5. Horizontal amplification factor for a PVC water pipe buried in sandy soil (A): (a) 13 magnitude; (b) wrapped phase; (c) unwrapped phase. The additional phase shift is marked by 14 the red arrow.

15 Fig. 6. Vertical amplification factor for a PVC water pipe buried in sandy soil (A): (a) 16 magnitude; (b) wrapped phase; (c) unwrapped phase. The additional phase shifts are marked 17 by the red arrow.

18 Fig. 7. Horizontal amplification factor for a PVC water pipe buried in clay Soil (B): (a) 19 magnitude; (b) unwrapped phase.

20 Fig. 8. Vertical amplification factor for a PVC water pipe buried in clay soil (B): (a) 21 magnitude; (b) phase.

22 Fig. 9. Effect of the burial depth on the horizontal amplification factor for sandy soil (A): for 23 magnitude plots, the burial depth are (a) $0.5 \mathrm{~m}$; (b) $1 \mathrm{~m}$; (c) $1.5 \mathrm{~m}$; for phase plots, the burial 24 depth are (d) $0.5 \mathrm{~m}$; (e) $1 \mathrm{~m}$; (f) $1.5 \mathrm{~m}$.

25 Fig. 10. Effect of the burial depth on the vertical amplification factor for sandy soil (A): for 
1 magnitude plots, the burial depth are (a) $0.5 \mathrm{~m}$; (b) $1 \mathrm{~m}$; (c) $1.5 \mathrm{~m}$; for phase plots, the burial

2 depth are (d) $0.5 \mathrm{~m}$; (e) $1 \mathrm{~m}$; (f) $1.5 \mathrm{~m}$.

3 Fig. 11. Pipe rig layout.

$4 \quad$ Fig. 12. Shaker mounted on the plastic end-plate.

$5 \quad$ Fig. 13. Measurement setup showing geophones and PVDF wires.

6 Fig. 14. Pipe wall and ground surface displacements: (a) magnitude; (b) unwrapped phase.

$7 \quad$ Fig. 15 . Real part of the $s=1$ wavenumber.

8 Fig. 16. Magnitude and phase of horizontal amplification factor, theory and experiment.

9 Fig. 17. Magnitude and phase of vertical amplification factor, theory and experiment. 


\section{List of table captions}

2 Table 1 Elastic waves in representative soils

3 Table 2 Properties of the pipe, soil and water

4 Table 3 Geometrical and material properties of the pipe and soil

5 Table 4 Bulk Properties of the soil 


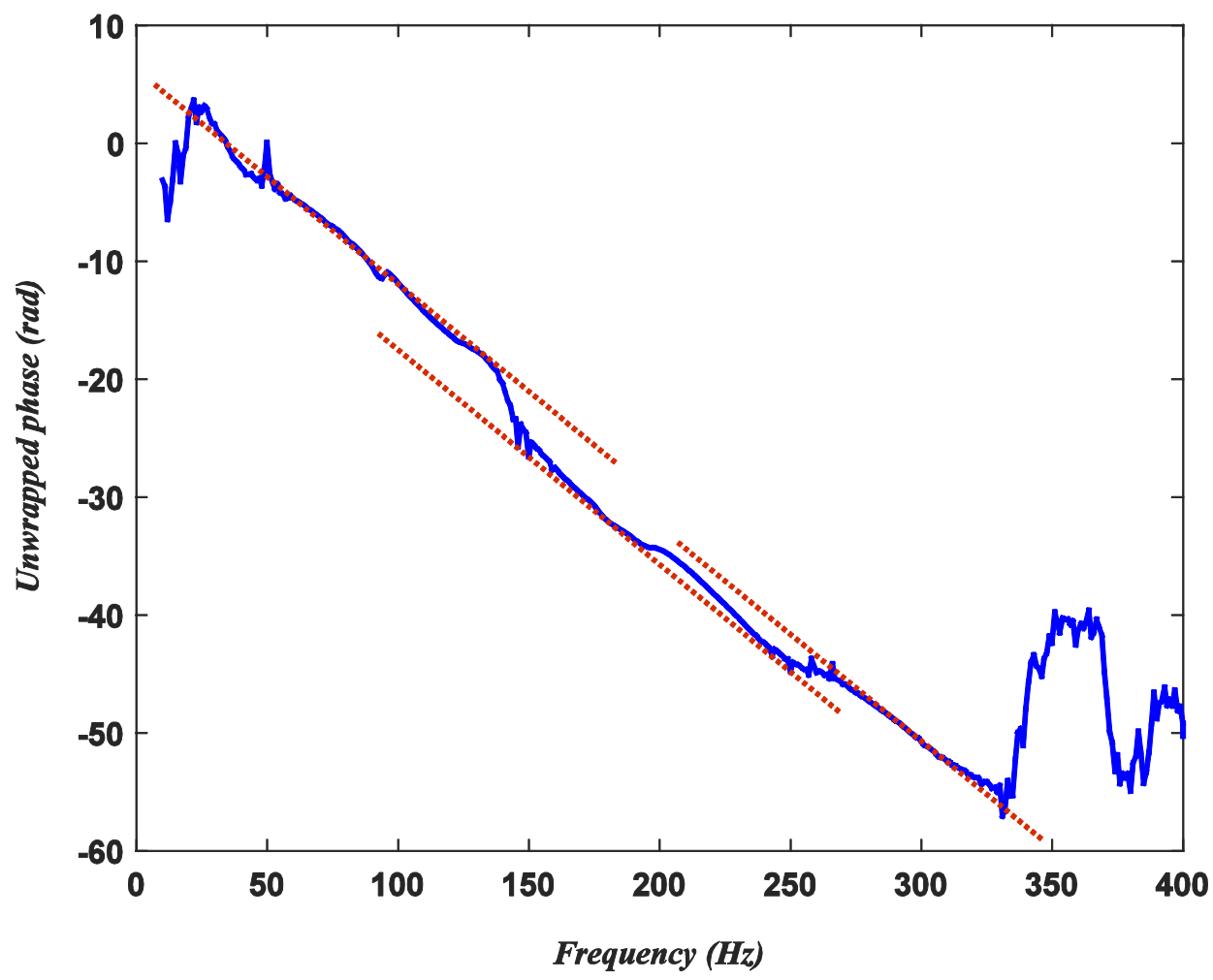

Fig. 1. Plot of the unwrapped phase measured by geophones showing abrupt phase changes between straight line sections [11]. 


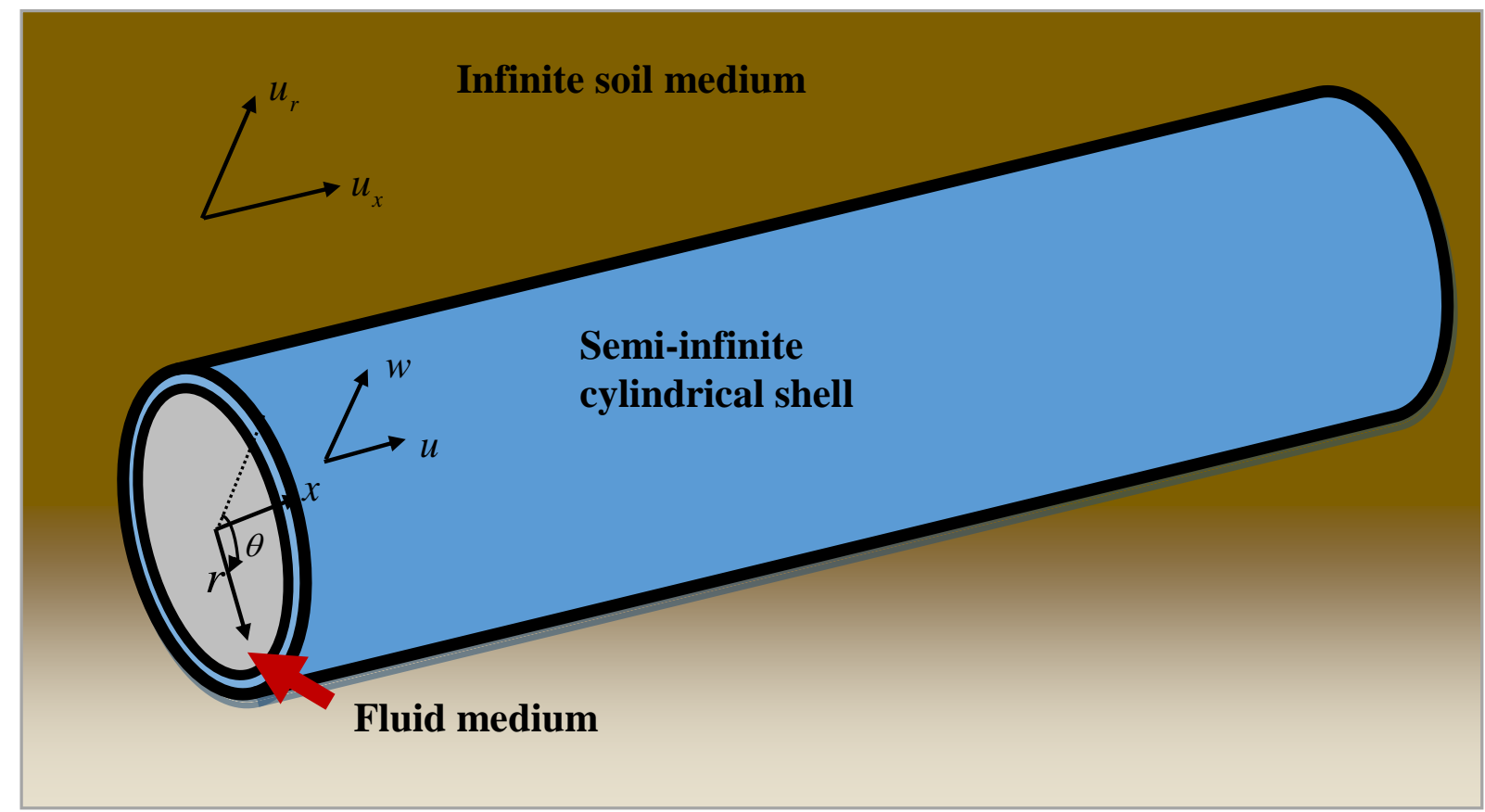

Fig. 2. The cylindrical co-ordinate system for a fluid-filled pipe surrounded by an infinite elastic medium. 


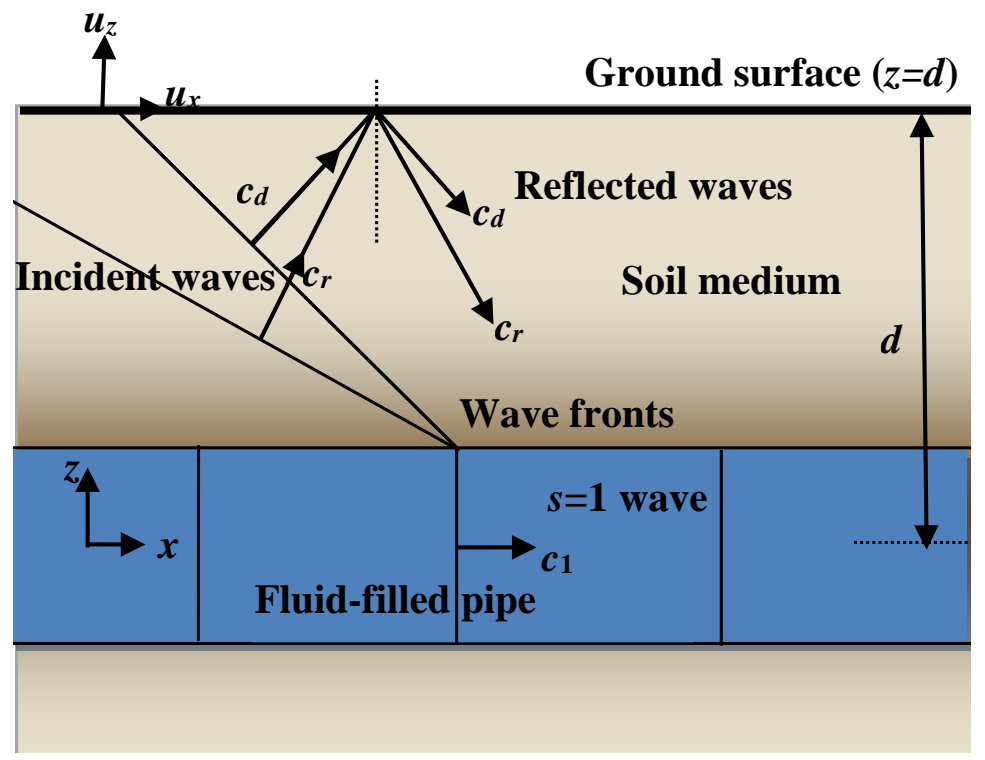

Fig. 3. Illustration of reflection of the radiated elastic waves induced by the fluid-borne wave motion in the Cartesian co-ordinate $(x, y, z)$ system. $c_{1}, c_{d}$ and $c_{r}$ denote the phase velocity of the $s=1$ wave, compressional and shear velocities in the soil respectively. 
(a)

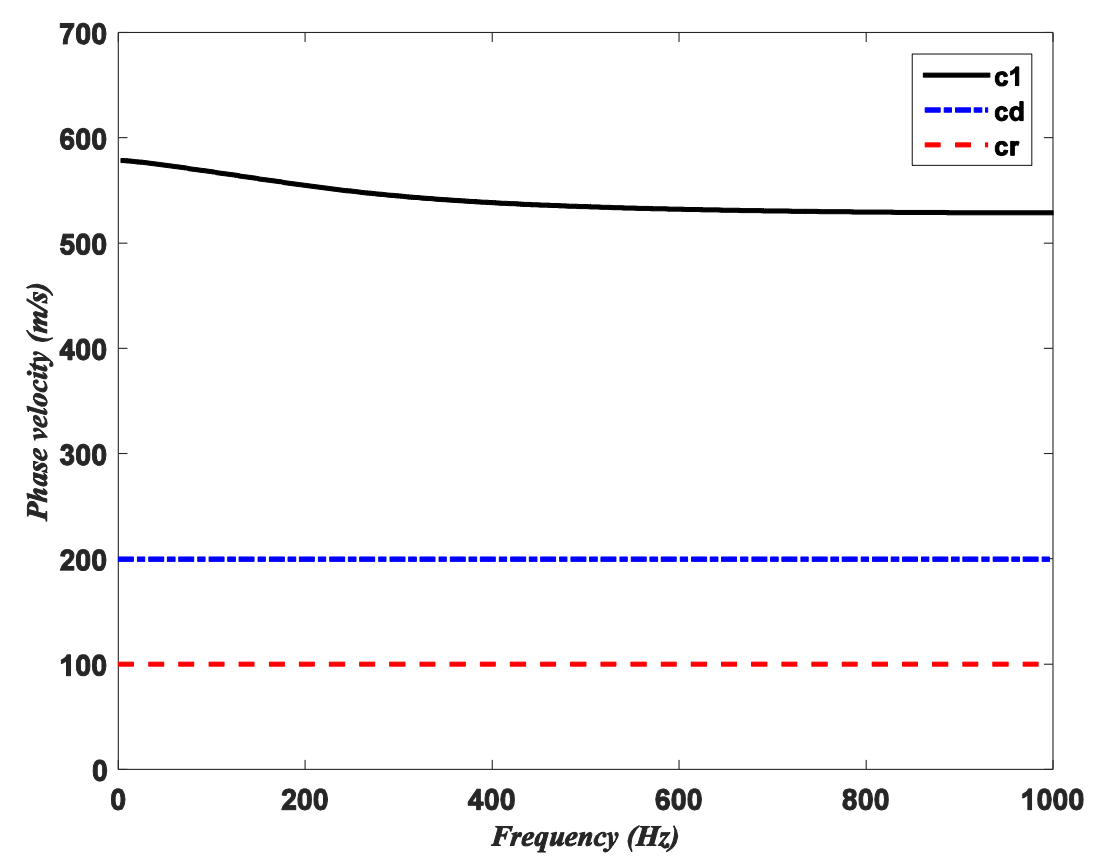

(b)

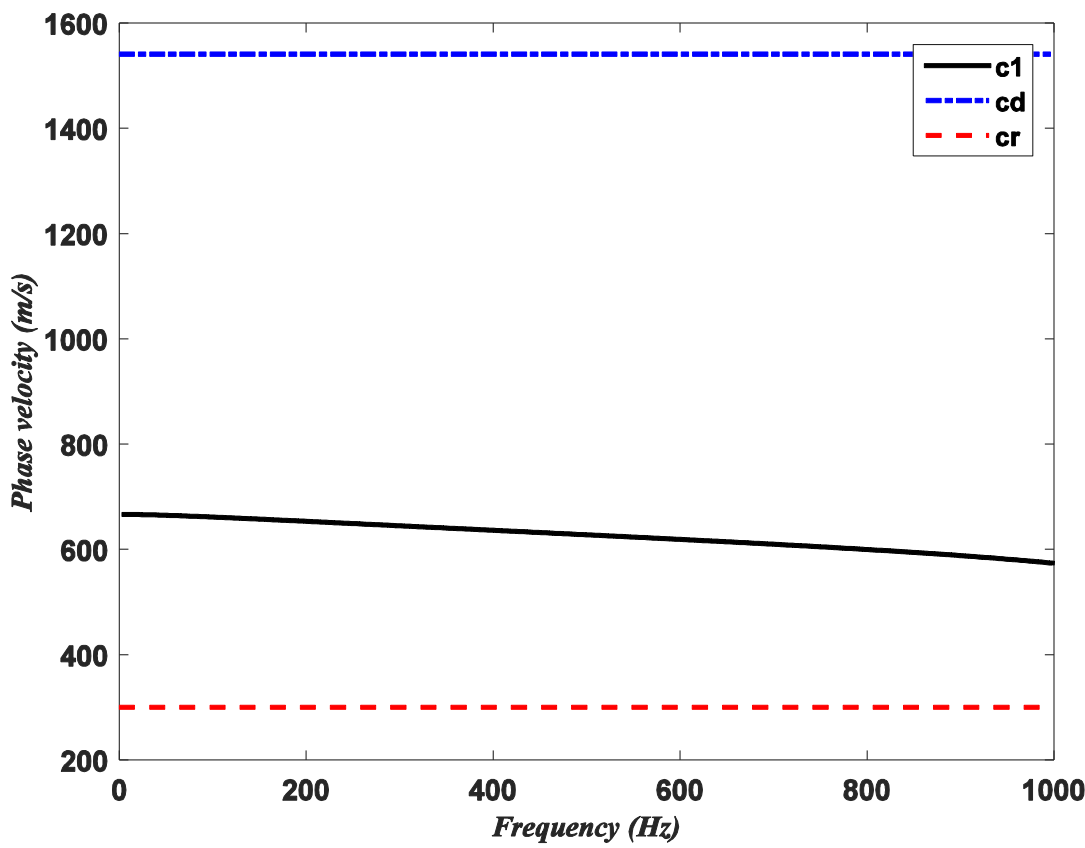

Fig. 4. Phase velocities of the $s=1$ wave for a PVC water pipe buried in: (a) sandy soil A and (b) clay soil (B) in comparison with the compressional $\left(c_{d}\right)$ and shear velocities $\left(c_{r}\right)$ in the surrounding soil. 
(a)

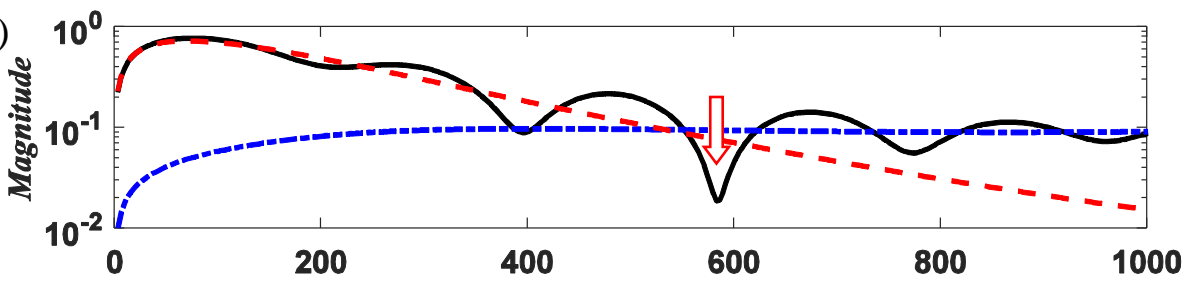

(b)

(c)
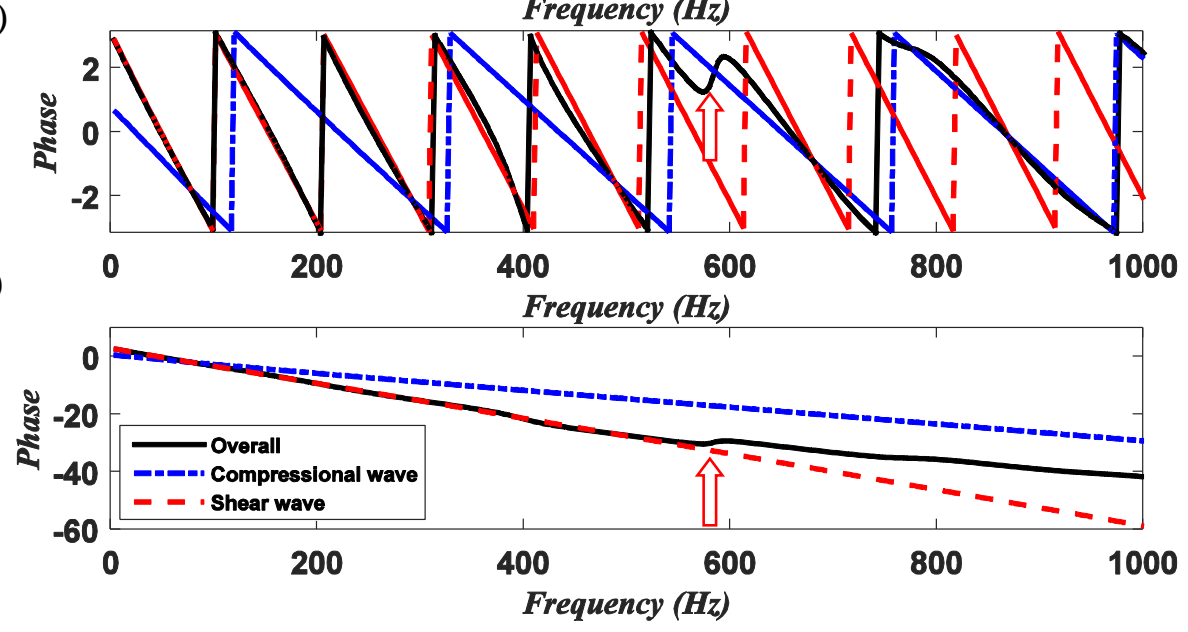

Fig. 5. Horizontal amplification factor for a PVC water pipe buried in sandy soil (A): (a) magnitude; (b) wrapped phase; (c) unwrapped phase. The additional phase shift is marked by the red arrow. 


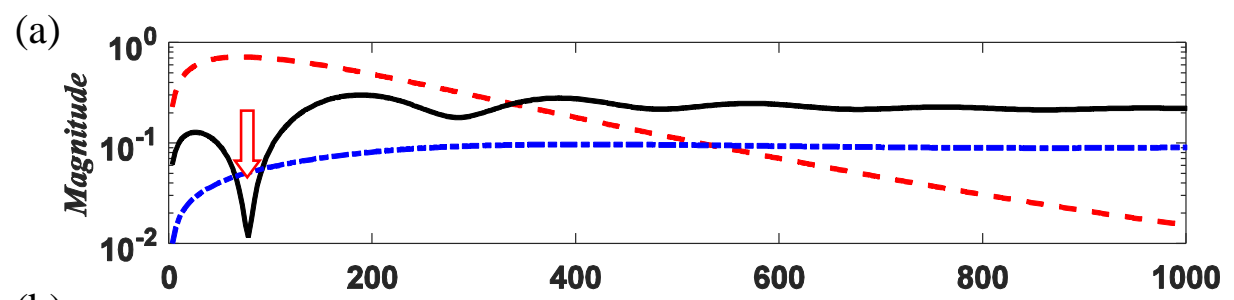

(b)
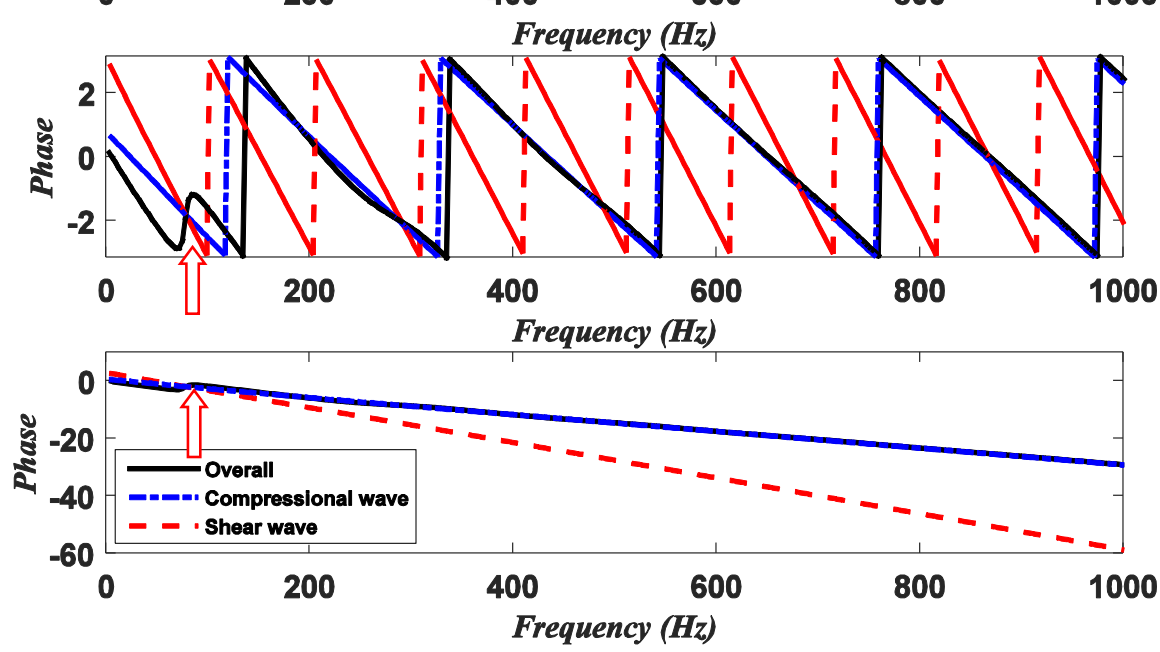

Fig. 6. Vertical amplification factor for a PVC water pipe buried in sandy soil (A): (a) magnitude; (b) wrapped phase; (c) unwrapped phase. The additional phase shifts are marked by the red arrow. 
(a)

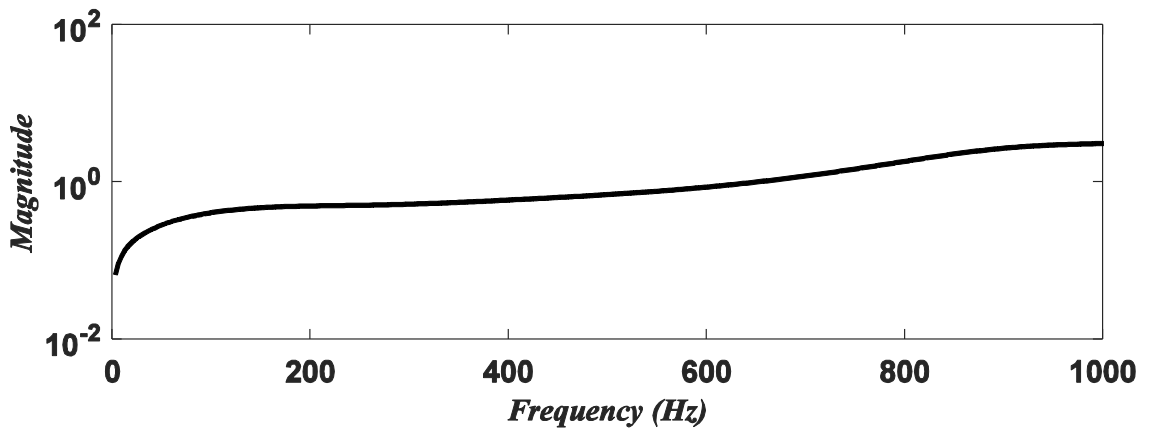

(b)

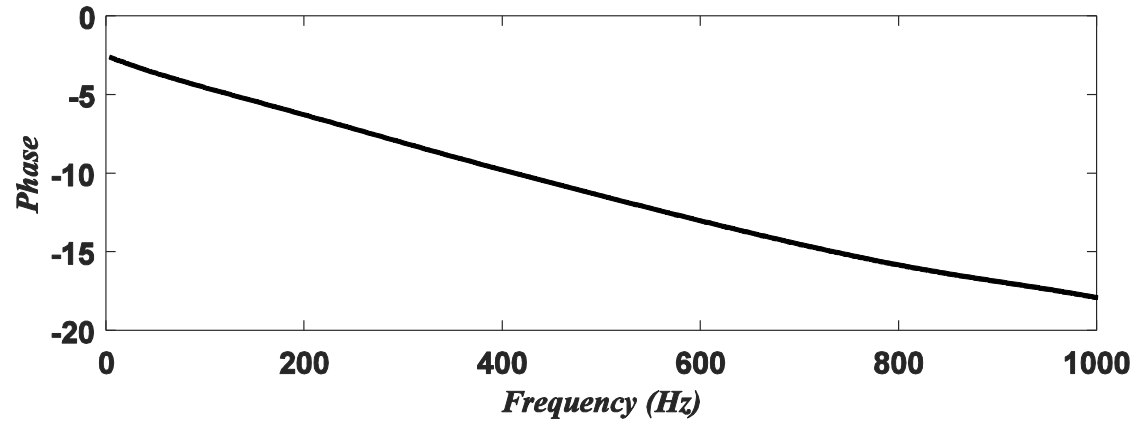

Fig. 7. Horizontal amplification factor for a PVC water pipe buried in clay Soil (B): (a) magnitude; (b) unwrapped phase. 
(a)

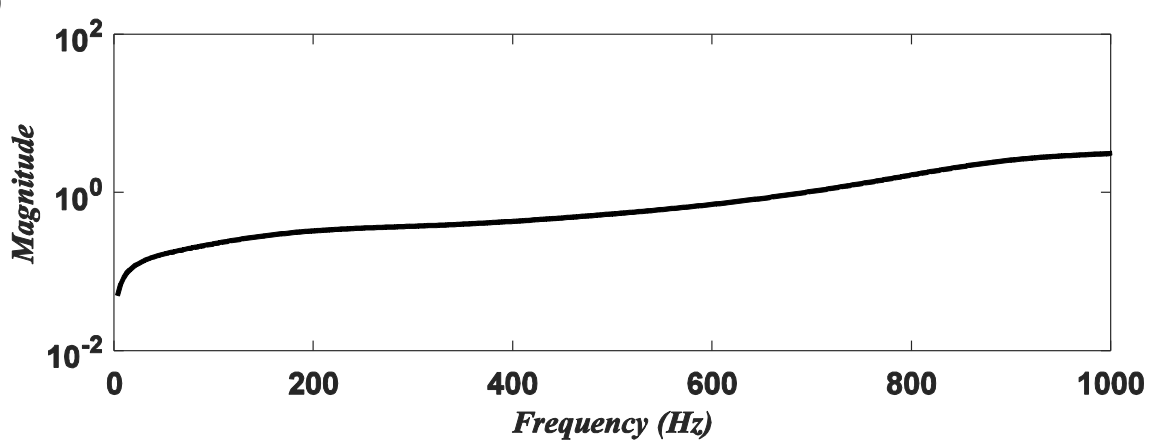

(b)

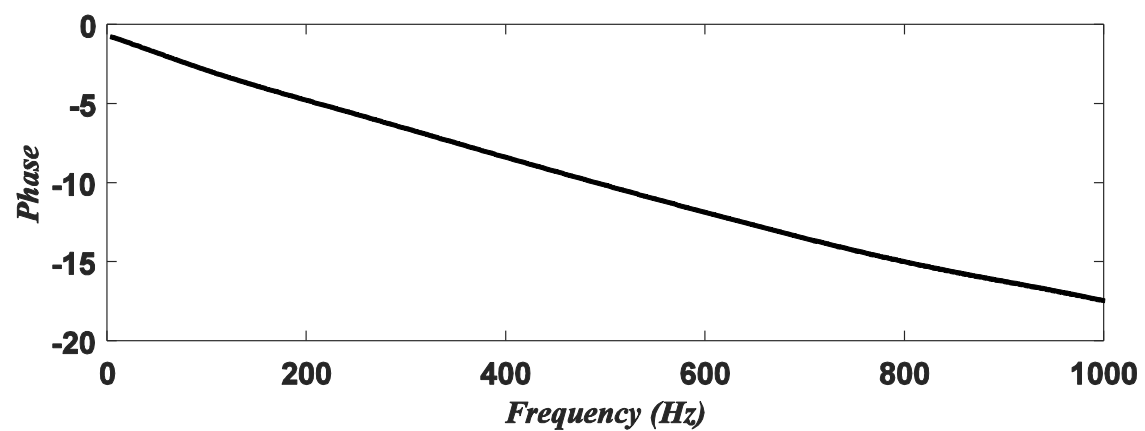

Fig. 8. Vertical amplification factor for a PVC water pipe buried in clay soil (B): (a) magnitude; (b) phase. 

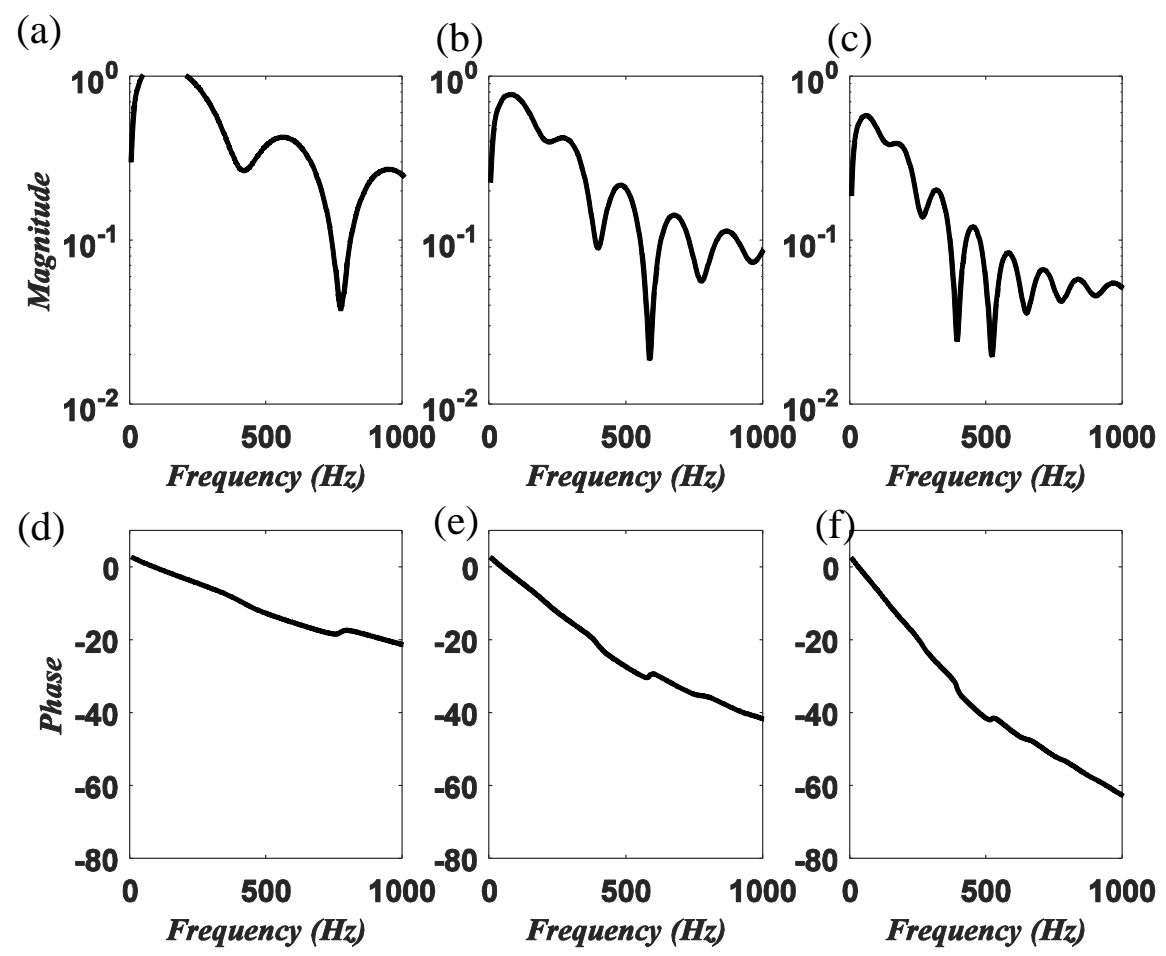

Fig. 9. Effect of the burial depth on the horizontal amplification factor for sandy soil (A): for magnitude plots, the burial depth are (a) $0.5 \mathrm{~m}$; (b) $1 \mathrm{~m}$; (c) $1.5 \mathrm{~m}$; for phase plots, the burial depth are (d) $0.5 \mathrm{~m}$; (e) $1 \mathrm{~m}$; (f) $1.5 \mathrm{~m}$. 

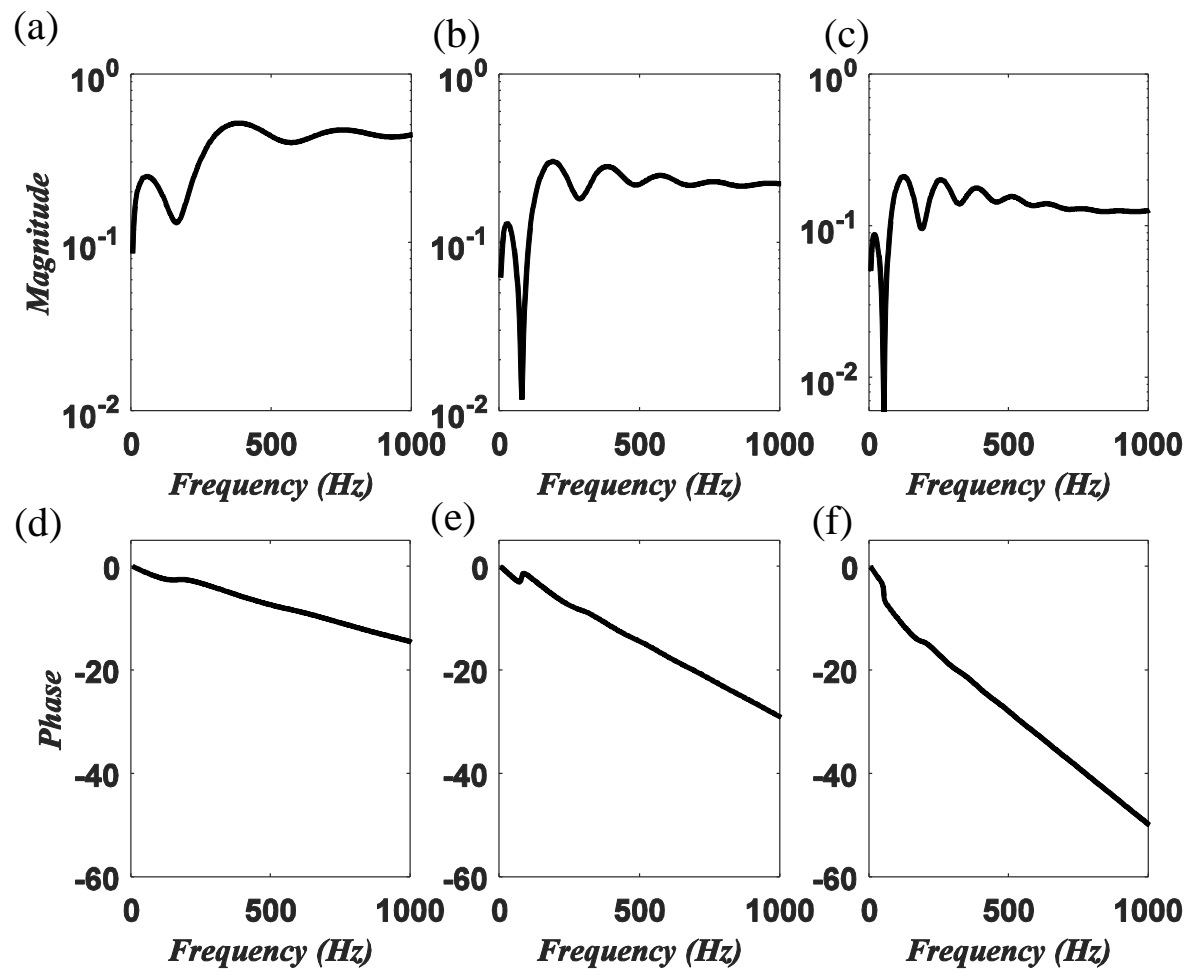

(e)

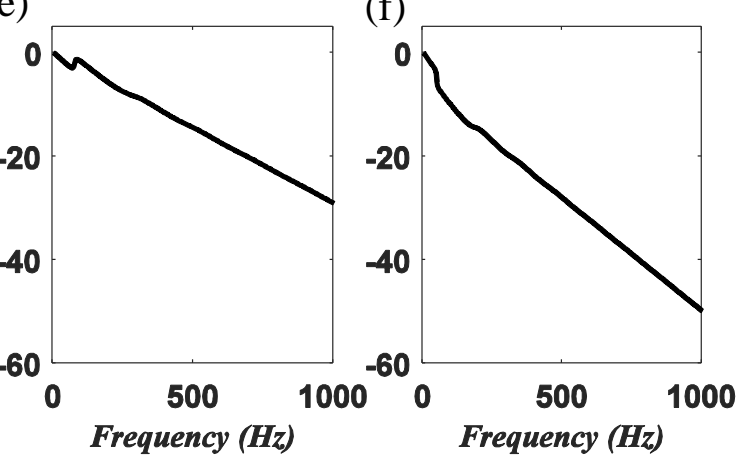

Fig. 10. Effect of the burial depth on the vertical amplification factor for sandy soil (A): for magnitude plots, the burial depth are (a) $0.5 \mathrm{~m}$; (b) $1 \mathrm{~m}$; (c) $1.5 \mathrm{~m}$; for phase plots, the burial depth are (d) $0.5 \mathrm{~m}$; (e) $1 \mathrm{~m}$; (f) $1.5 \mathrm{~m}$. 


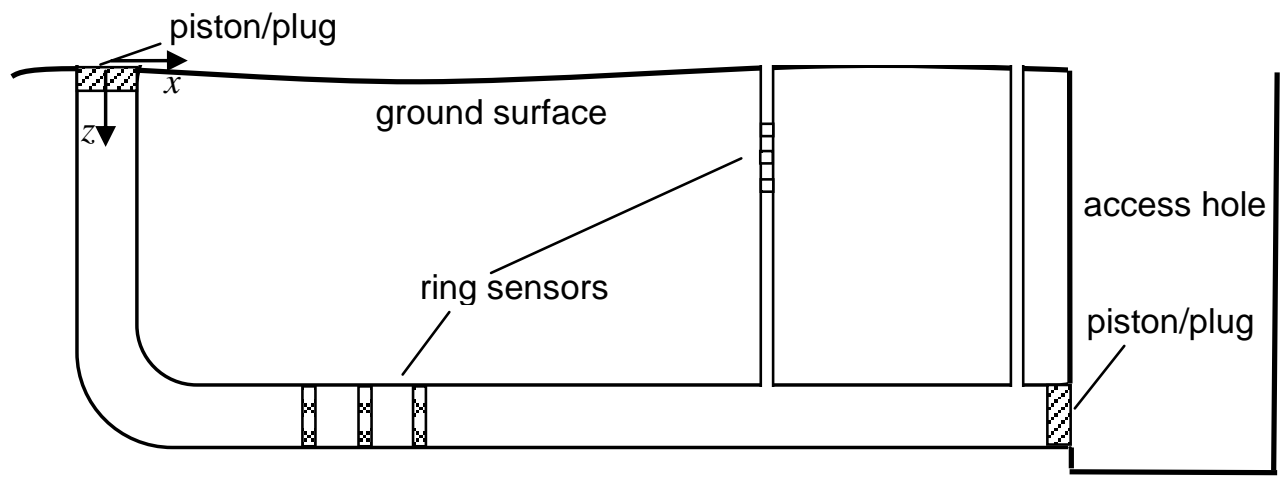

Fig. 11. Pipe rig layout. 


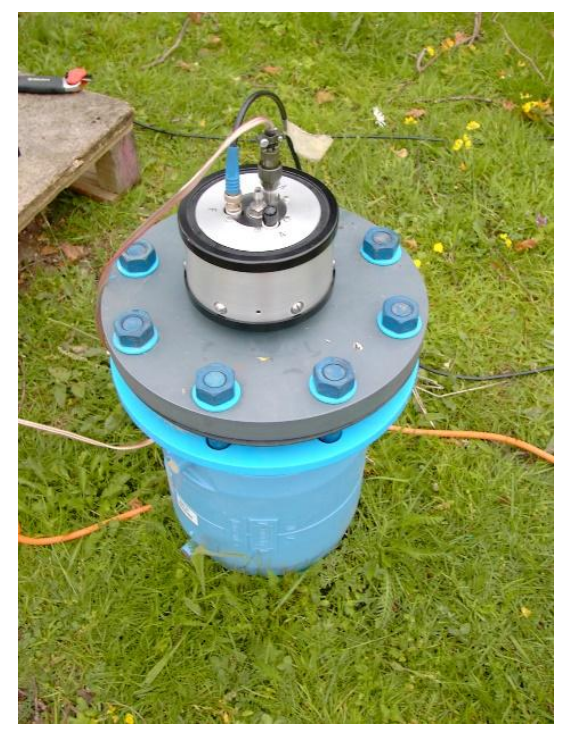

Fig. 12. Shaker mounted on the plastic end-plate. 


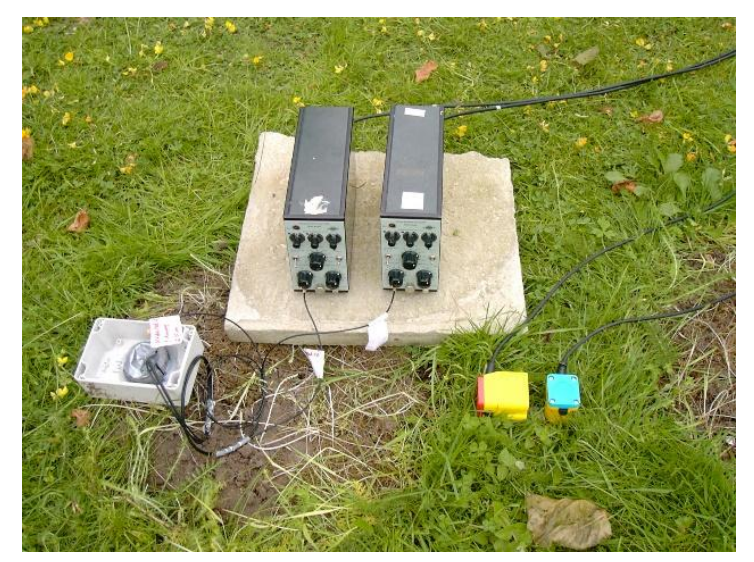

Fig. 13. Measurement setup showing geophones and PVDF wires. 
(a)

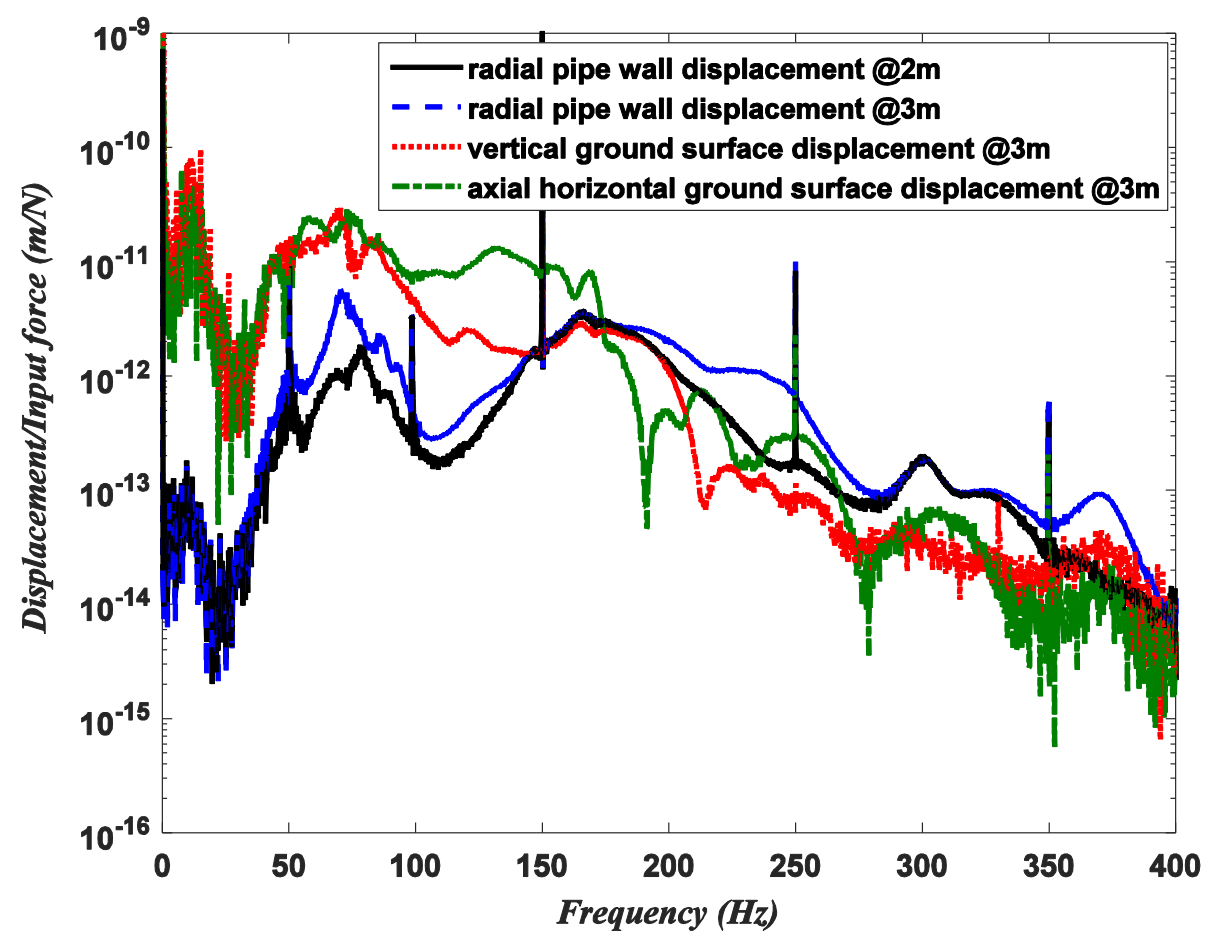

(b)

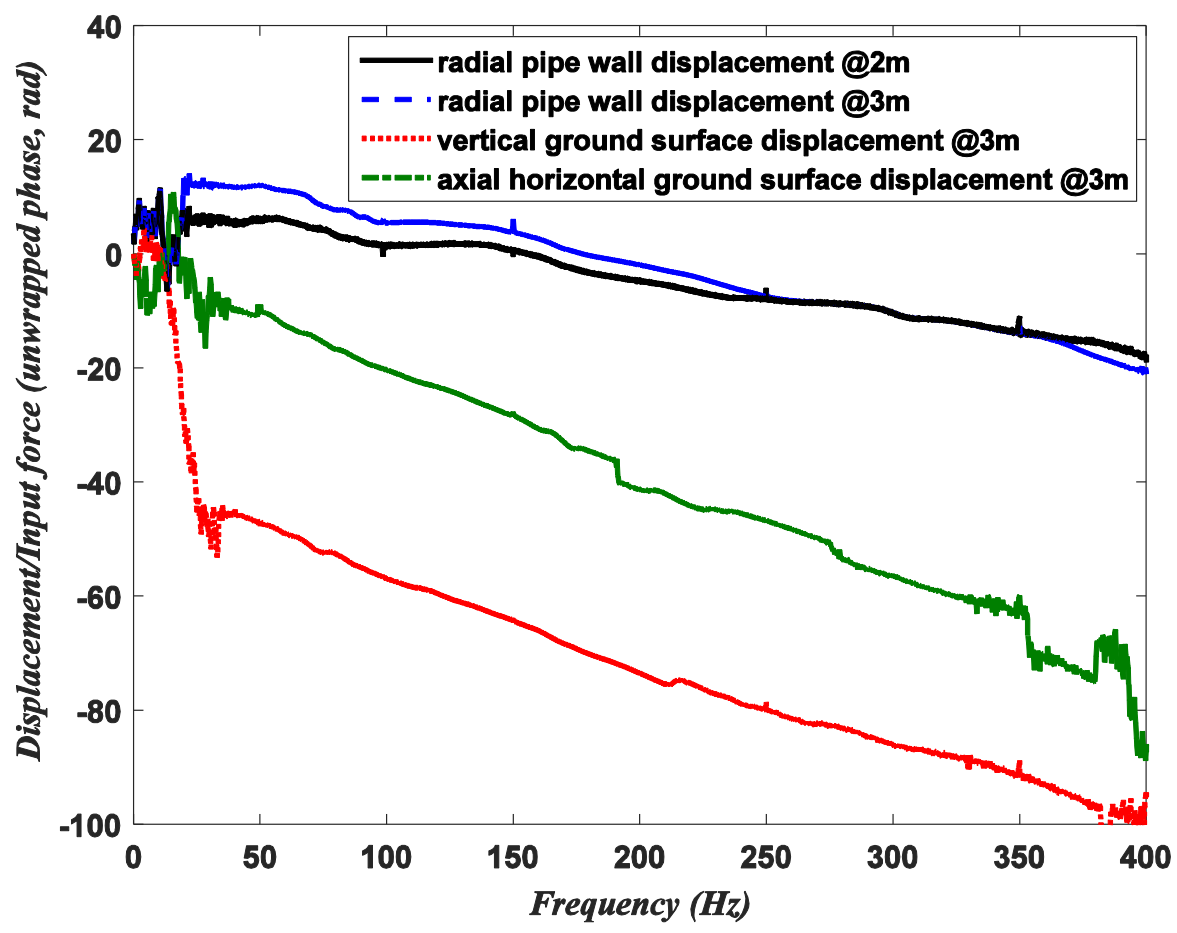

Fig. 14. Pipe wall and ground surface displacements: (a) magnitude; (b) unwrapped phase. 


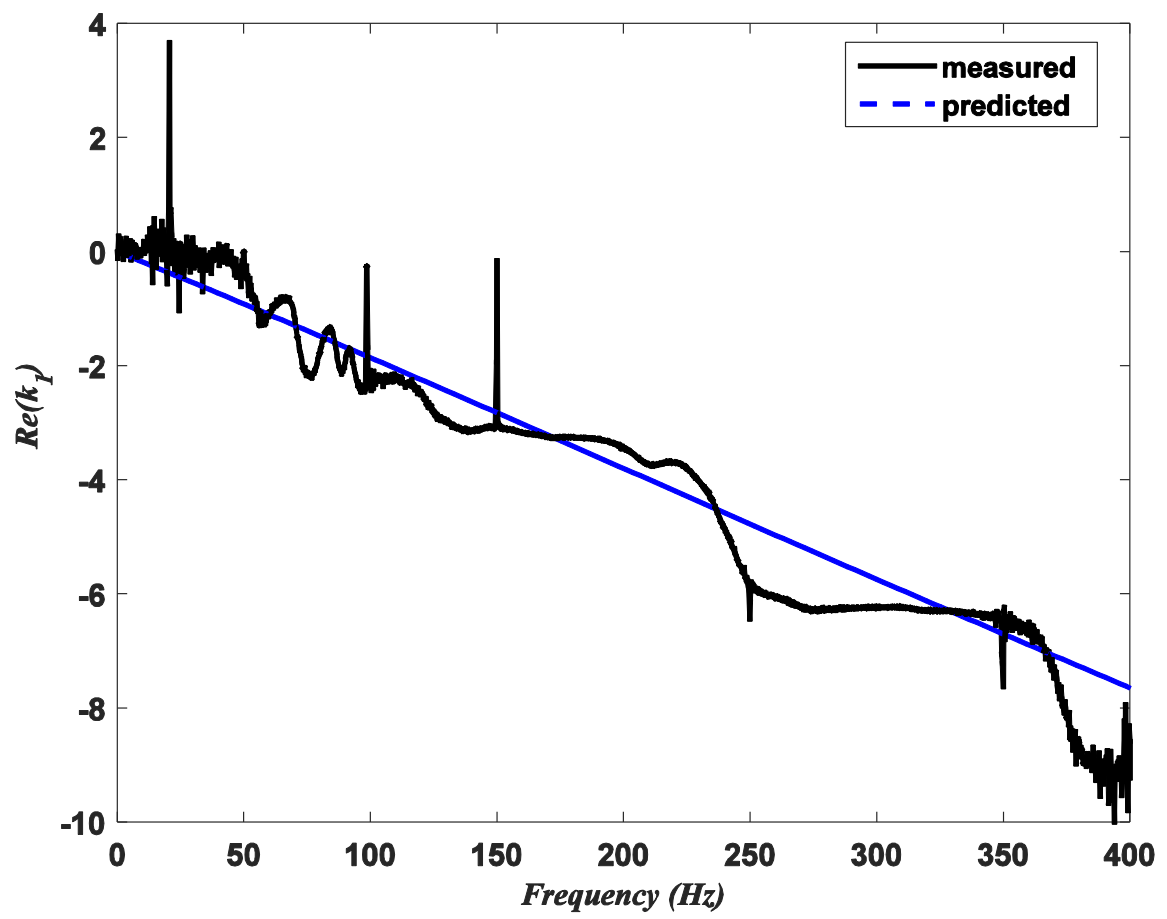

Fig. 15. Real part of the $s=1$ wavenumber. 

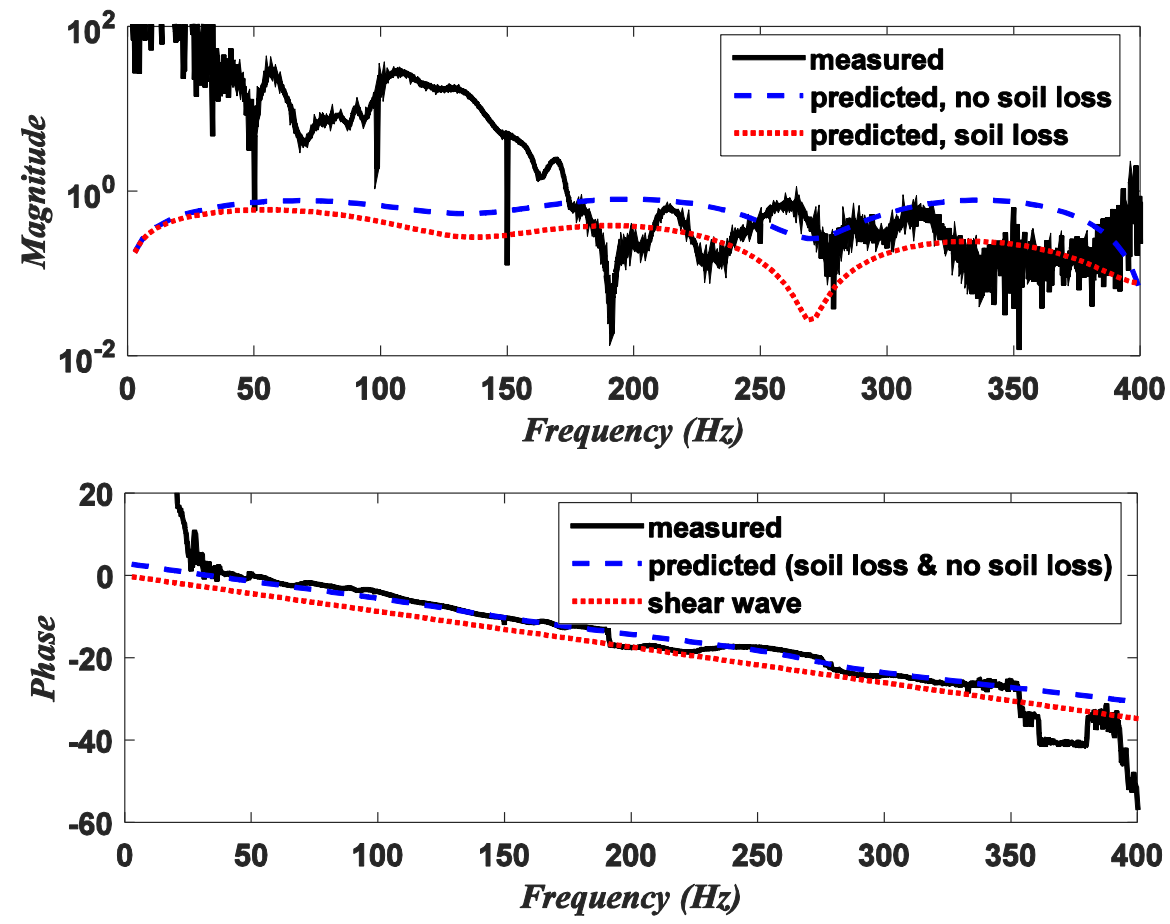

Fig. 16. Magnitude and phase of horizontal amplification factor, theory and experiment. 

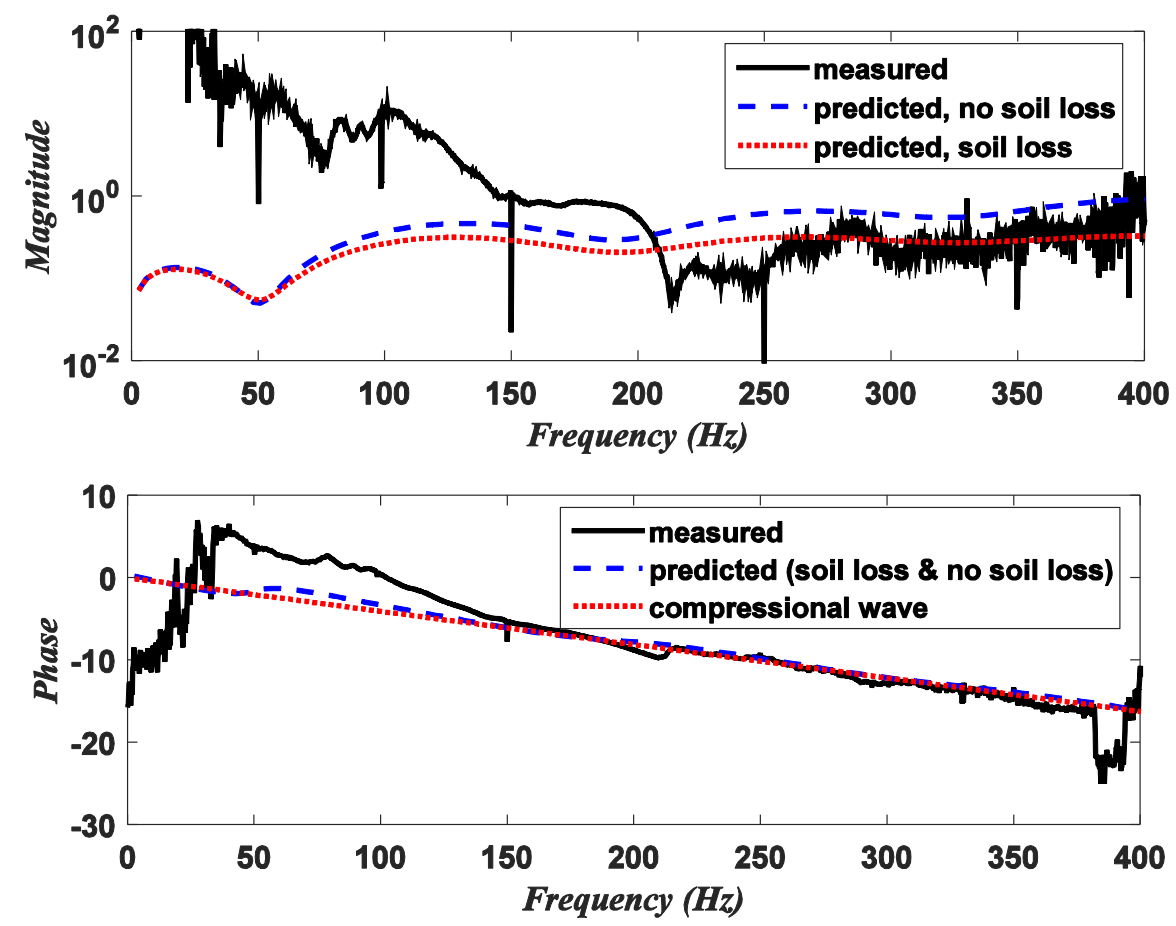

Fig. 17. Magnitude and phase of vertical amplification factor, theory and experiment. 
Table 1 Elastic waves in representative soils

\begin{tabular}{lll}
\hline & Sandy soil & Clay soil \\
\hline Phase velocity & $c_{1}>c_{d}$ or $\operatorname{Re}\left(k_{1}^{2}\right)<\operatorname{Re}\left(k_{d}^{2}\right)$ & $c_{d}>c_{1}>c_{r}$ or \\
(wavenumber) & $c_{1}>c_{r}$ or $\operatorname{Re}\left(k_{1}^{2}\right)<\operatorname{Re}\left(k_{r}^{2}\right)$ & $\operatorname{Re}\left(k_{d}^{2}\right)<\operatorname{Re}\left(k_{1}^{2}\right)<\operatorname{Re}\left(k_{r}^{2}\right)$ \\
Badiation & Both compressional and & \\
& shear waves radiate. & Shear wave radiates only. \\
\hline
\end{tabular}


Table 2 Properties of the pipe, soil and water

\begin{tabular}{lllll}
\hline Properties & PVC & Soil A & Soil B & water \\
& & & & \\
\hline Density $\left(\mathrm{kg} / \mathrm{m}^{3}\right)$ & 2000 & 2000 & 2000 & 1000 \\
Young's modulus $\left(\mathrm{GN} / \mathrm{m}^{2}\right)$ & 5.0 & - & - & - \\
Bulk's modulus $\left(\mathrm{GN} / \mathrm{m}^{2}\right)$ & - & 0.053 & 4.5 & 2.25 \\
Shear modulus $\left(\mathrm{GN} / \mathrm{m}^{2}\right)$ & 1.79 & 0.02 & 0.18 & - \\
Poisson's ratio & 0.4 & - & - & - \\
Material loss factor & 0.065 & 0.1 & 0.1 & - \\
\hline
\end{tabular}


Table 3 Geometrical and material properties of the pipe and soil

\begin{tabular}{ll}
\hline Properties & MDPE Pipe \\
\hline Mean radius $(\mathrm{m})$ & 0.0845 \\
Wall thickness $(\mathrm{m})$ & 0.011 \\
Density $\left(\mathrm{kg} / \mathrm{m}^{3}\right)$ & 900 \\
Young's modulus $\left(\mathrm{GN} / \mathrm{m}^{2}\right)$ & 2.0 \\
Shear modulus $\left(\mathrm{GN} / \mathrm{m}^{2}\right)$ & - \\
Poisson's ratio & - \\
Material loss factor & 0.06 \\
\hline
\end{tabular}


Table 4 Bulk Properties of the soil

\begin{tabular}{ll}
\hline Properties & Soil \\
\hline Density $\left(\mathrm{kg} / \mathrm{m}^{3}\right)$ & 2000 \\
Poisson's ratio & 0.33 \\
Shear modulus $\left(\mathrm{GN} / \mathrm{m}^{2}\right)$ & 10 \\
Material loss factor & $0 / 0.1$ \\
Shear wavespeed $(\mathrm{m} / \mathrm{s})$ & $\sim 70$ \\
Compressional wavespeed $(\mathrm{m} / \mathrm{s})$ & $\sim 140$ \\
\hline
\end{tabular}

